\title{
Development and validation of a fully predictive high-fidelity simulation approach for predicting coarse road dynamic tire/road rolling contact forces
}

\author{
Daniel De Gregoriis ${ }^{\mathrm{a}, \mathrm{b}, *}$, Frank Naets ${ }^{\mathrm{b}, \mathrm{c}}$, Peter Kindt $^{\mathrm{a}}$, Wim Desmet ${ }^{\mathrm{b}, \mathrm{c}}$ \\ ${ }^{a}$ Vibration Mechanics, Goodyear Innovation Center* Luxembourg, Avenue Gordon Smith, \\ L-7750 Colmar-Berg, Luxembourg \\ ${ }^{b} K U$ Leuven, Department of Mechanical Engineering, Celestijnenlaan 300 B, B-3001, \\ Heverlee, Belgium \\ ${ }^{c}$ DMMS Lab, Flanders Make
}

\begin{abstract}
In this work, a fully predictive high-fidelity numerical approach for the simulation of a car tire rolling with a constant angular velocity over a coarse road surface is presented. A fully nonlinear vibro-acoustic finite element tire model is combined with an Arbitrary Lagrangian Eulerian formulation to describe both the rolling dynamics as well as the interaction between the tire and coarse road surface. A geometrical constraint approach is used to describe the tire/road interaction rather than a constitutive approach in order to keep the proposed method fully predictive, i.e. to not rely on measurement data. As both the use of a nonlinear finite element tire model, as well as the use of the geometrical constraint approach result in large computational costs and simulation times, the nonlinear Multi-Expansion Modal Reduction hyper-reduction method is applied. Numerical costs and corresponding simulation times are reduced by a factor of order of magnitude 100 (from several months to hours), therefore making the proposed approach feasible to use in an industrial design and development process. Two alternative linearized approaches, based on the proposed nonlinear approach, are considered as well. While the proposed nonlinear approach shows a good, consistent qualitative and quantitative correspondence between experimental and numerical simulation results for the entire $0-400 \mathrm{~Hz}$ frequency range of interest, with normalized Power Spectral Density differences between 0.1 and $0.2 \mathrm{~dB}($ ref. $1 \mathrm{~N} / \mathrm{Hz})$, the linearized approaches are limited to lower frequency ranges of $0-85 \mathrm{~Hz}$ and $0-200 \mathrm{~Hz}$ and are therefore not suitable for the intended application. The overall performance of the proposed nonlinear approach confirms its use as a possible alternative to standard time-consuming experimental approaches.
\end{abstract}

Keywords: Dynamic tire/road rolling contact simulation, Nonlinear finite

\footnotetext{
${ }^{*}$ Corresponding author

Email address: daniel.degregoriis@kuleuven.be (Daniel De Gregoriis)
} 
element tire model, Tire/road interior noise

2010 MSC: 00-01, 99-00

\section{Introduction}

Tire/road noise is a major source of vehicle noise in modern cars (both those equipped with an internal combustion engine and electrical motor), and therefore is an important contribution to road traffic noise [1]. This has resulted 5 in vehicle and tire manufacturers refining their car and tire designs to help reduce traffic noise. Typically over 50 different performance criteria have to be taken into account when designing and optimizing a passenger car tire design. These are related to (but not limited to) energy efficiency, handling, wear and noise. Due to the complex structure of a typical passenger car tire, most of 10 these criteria are coupled: trying to enhance one performance may affect other performances. For example, increasing the damping characteristics of the tire tread rubber compound will typically reduce tire/road noise, but will also affect other performance areas such as handling and energy efficiency. Traditionally, an experimental approach has been used during the tire design process to as15 sess the different performance areas of a specific tire design. This approach has drawbacks in terms of costs of running the experiments, as well as time needed to build several physical tires. In order to cope with the increasing need to optimize multiple tire performances simultaneously, as well as shorter product development times, predictive numerical simulation techniques could be used, ${ }_{20}$ rather than time-consuming experiments. A numerical approach offers the possibility to perform virtual assessments of different tire designs in a more efficient and cost-effective manner than building and testing physical tires.

In this work, the prediction of structure-borne interior noise due to a tire rolling over a coarse road surface is considered. More specifically, the dynamic

25 vertical hub forces, resulting from the tire/road contact forces, are considered as they excite the vehicle suspension and result in the structure-borne interior noise. The structure-borne noise due to tire/road interaction tends to be dominant over airborne noise inside the passenger cabin below $400 \mathrm{~Hz}$ [1. Being able to predict the structure-borne noise of a specific tire design is therefore valuable in an overall performance assessment during virtual tire development.

Several approaches to numerically predict the structure-borne tire/road noise have been suggested in literature. An extensive overview can be found in the works by Hoever [2] and Winroth [3]. The numerical approaches to predict tire/road noise typically consist of a mathematical tire structure model and a

35 tire/road contact model. The tire models used in the numerical approaches can generally be divided into two categories: low-order analytical models (analytical and semi-analytical formulations incorporating the necessary physics) and higher-order numerical models (physics-based discretized continuum descriptions). The low-order analytical models tend to be based on analytical 40 descriptions and physical insights, yielding computationally efficient numerical models that can be evaluated in a fast and efficient manner. Examples of these 
models can be found in the works by Graham [4, Kindt et al. [5, Kropp [6], Larsson et al. 7], Pinnington [8, 9] and Sabiniarz and Kropp [10], amongst others. However, linking the parameters of these models to physical tire design 45 parameters, such as reinforcement fiber angles, tire cross-section geometry, rubber compound properties etc., is typically not possible. Furthermore, inherent nonlinear tire behavior (due to e.g. the rubber compounds and reinforcement materials) is typically not included in these models, unless the models are tuned to a specific configuration of a physical tire using measurements. Therefore, the 50 use of low-order analytical models in a purely predictive numerical design optimization approach is not feasible. The use of higher-order numerical tire models, where the models are built in a rigorous, mathematical way analogous to the real physical tire, appears to be more appropriate. Physical design parameters, such as e.g. reinforcement fiber angles, are typically mapped one-to-one to the 55 numerical model parameters. Different material properties can be described by dedicated constitutive models, using material test data rather than systemlevel tire measurements. The detailed tire geometry can be replicated, given an adequate discretization of the tire cross-section and circumference. These highfidelity models are needed in a predictive numerical design approach. These ${ }_{60}$ numerical tire models are typically described using a nonlinear finite element (FE) formulation. Using a FE formulation allows to include all sorts of nonlinearities, thereby enabling the inclusion of a range of relevant physical effects such as nonlinear large strain behavior, incompressible material behavior, time and/or frequency dependent viscous material behavior, embedded reinforcement 65 behavior etc. Examples of the use of high-fidelity FE tire models to predict tire/road interaction can be found in the works by e.g. Brinkmeier et al. 11], Lopez Arteaga [12] and Nackenhorst [13].

A specific aspect for predictive tire simulation is the description of the contact between the tire tread and the road surface. This is typically done

70 through a constitutive approach, yielding contact stresses in function of the displacements of the interacting bodies in their interface layer, or a geometrical constraint-based approach can be adopted [14, Chapter 5]. While the constitutive approach typically yields more accurate results [14, Chapter 5.1.2], it also requires measurement data to derive the specific constitutive laws, which

75 depend on the problem being studied and its corresponding coupled boundary conditions. In the geometrical constraint-based approach, non-penetration constraints are added to the system equations. These constraints are then imposed via either the Lagrange-multiplier method, the penalty method, or combinations of both [14, Chapter 6]. Typically, the geometrical-constraint based approach leads to less accurate results, as it neglects smaller-scale contact phenomena 14. Chapter 5.1.1]. The geometric approach is also susceptible to a range of issues resulting from overly coarse meshes [2, 14, 15, 16], leading to very dense meshes and associated high computational loads. This issue is inherently circumvented through the constitutive contact approach, which provides a more generalized 85 nonlinear contact force definition. Many authors have therefore proposed methods in this framework $2,3,6,11,12,15,17,18,19,20,21$. Even though these are generally powerful methods, the contact model parameters typically require 
tuning on experimental data, making them less suitable for predictive modeling.

To the authors' knowledge, a fully predictive high-order numerical approach has not been proposed in literature before for general tire design purposes, as a result of the infeasible computational load or required experimental model updating. However, for accurate design analysis this is approach is highly valuable. Therefore, in this work a predictive high-fidelity numerical approach for the calculation of tire/road contact forces during rolling with a constant angular

95 velocity over a coarse road surface is proposed. The proposed approach consists of three main stages:

- A high-fidelity nonlinear vibro-acoustic FE tire model, in combination with an Arbitrary Lagrangian Eulerian (ALE) formulation [22] is used to describe both the rolling tire dynamics as well as the coarse road excitation. As coarse road excitation and structure-borne interior noise is considered in this work, the required approximation of a ribbed slick tire design is justified, as there is little to no contribution of the tread pattern induced structure-borne noise below $400 \mathrm{~Hz}$ [1]. This model is coupled in a vibro-acoustic fluid-structure interaction (FSI) for the air cavity and tire structure. To allow for predictive analysis, geometrical contact constraints are used, together with a dense mesh in the tire/road contact area.

- In order to limit the dynamic evaluation cost, a nonlinear projection-based model order reduction (MOR) technique, the Multi-Expansion Modal Reduction method (MEM) 223, 24, 25, is applied to the proposed predictive high-fidelity approach. This nonlinear MOR technique belongs to a class of hyper-reduction techniques for nonlinear structural FE problems [26]. Applying the proposed hyper-reduction method drastically reduces the numerical costs of the proposed high-fidelity approach, while still retaining a high level of accuracy with respect to the original numerical model. As is shown in this work, the reduction of numerical costs and associated computational time is key in the applicability of the proposed predictive approach. Two alternative linearized approaches derived from the proposed reduced fully nonlinear predictive approach are presented as well. These approaches are used to assess the need of the reduced fully nonlinear FE approach to obtain a good correspondence between the experimental and numerical results.

- The overall hyper-reduced, predictive high-fidelity nonlinear FE approach allows to perform a fully-predictive numerical assessment of a tire design, as an alternative to performing the equivalent experimental assessment using a physical prototype tire.

Based on this proposed framework, the remainder of this paper is structured as follows: the full order structural model describing the rolling of a (ribbed) slick tire with a constant angular velocity over a coarse road surface is discussed in section 2. Modeling the tire/road surface rolling interaction as geo130 metrical contact constraints in an ALE-framework is discussed in section 2.4 
The reduced order modeling based on the full order tire model, is discussed in section 3 Validation of the proposed fully predictive high-fidelity numerical approach using experimental data is discussed in section 4. Two alternative linearized approaches are discussed and compared with the reduced fully nonlinear FE approach in this section as well to demonstrate the benefits of including the tire nonlinearity towards higher frequency ranges. Finally, conclusions are given in section 5 .

\section{Full-order nonlinear finite element tire model}

This section summarizes the full order nonlinear FE model contributions 140 for assessing the dynamic tire behavior and tire spindle (hub) forces resulting from coarse road contact. First the coupled nonlinear vibro-acoustic equations are considered, next the contact modeling is presented, and finally the time integration is discussed.

\subsection{Nonlinear structural tire model}

The semi-discretized, i.e. discretized in space but continuous in time, nonlinear finite element equations of motion describing the structural dynamic response of a tire rolling with a constant angular velocity over a coarse road surface can be expressed as [11, 13]:

$$
\mathbf{M} \ddot{\mathbf{x}}+\mathbf{G}_{\mathbf{A L E}}(\mathbf{x}, \mathbf{w}) \dot{\mathbf{x}}+\mathbf{f}(\mathbf{x}, \dot{\mathbf{x}})-\mathbf{f}_{\mathrm{ALE}}(\mathbf{x}, \mathbf{w})=\mathbf{f}_{\mathbf{p}}\left(\mathbf{x}, p_{a}\right)+\mathbf{f}_{\mathbf{c}}\left(\mathbf{x}, \mathbf{x}_{\mathbf{r}}\right)+\mathbf{f}_{\mathrm{e}}
$$

Here, the current configuration $\mathbf{x}(t) \in \mathbb{R}^{n}$ is defined as:

$$
\mathbf{x}(t)=\mathbf{x}_{0}+\mathbf{u}(t)
$$

${ }_{145}$ Where $\mathbf{x}_{0}$ is a reference configuration and $\mathbf{u}(t)$ is the total displacement with respect to the reference configuration at time $t$. The first and second derivatives of the current configuration with respect to time are denoted as $\dot{\mathbf{x}}$ and $\ddot{\mathbf{x}}$, respectively. All configuration-dependent terms therefore depend on time as well, but this time-dependency is dropped from notation for clarity reasons. Furthermore, the constant system mass matrix is denoted as $\mathbf{M} \in \mathbb{R}^{n \times n}$, the configuration dependent gyroscopic matrix is denoted as $\mathbf{G}_{\mathbf{A L E}}(\mathbf{x}, \mathbf{w}) \in \mathbb{R}^{n \times n}$, where $\mathbf{w}$ is the guiding velocity, and the configuration dependent gyroscopic force vector is denoted as $\mathbf{f}_{\mathbf{A L E}}(\mathbf{x}, \mathbf{w}) \in \mathbb{R}^{n}$. These terms are further discussed in more detail in section 2.2. The internal force vector is denoted by

$155 \mathbf{f}(\mathbf{x}, \dot{\mathbf{x}}) \in \mathbb{R}^{n}$ and includes nonlinear strain-displacement behavior and nonlinear nearly-incompressible (visco-) hyperelastic constitutive behavior, which describes e.g. the rubber compound behavior and reinforcement material behavior. In order to describe the nearly-incompressible behavior of the (visco)hyperelastic constitutive models, a mixed displacement-pressure $(\mathrm{u} / \mathrm{p})$ formulation is used [27, Chapter 6.4]. Details on the weak form of the internal force term and its discretization can be found in e.g. the works by Bathe [27, Chapter 6] and de Borst et al. [28, Chapter 3]. The inflation pressure forces acting on the 
boundary $\partial \Omega_{p}$ of the tire subdomain $\Omega_{p}$ are modeled as configuration-dependent follower forces, denoted by $\mathbf{f}_{\mathbf{p}}\left(\mathbf{x}, p_{a}\right) \in \mathbb{R}^{n}$. Here $p_{a}$ is the applied inflation air pressure. Information on its weak form and discretization can be found in e.g. Zienkiewicz et al. 29, Chapter 7.4]. The tire/road contact forces acting on the boundary $\partial \Omega_{c}$ of the tire subdomain $\Omega_{c}$ are denoted by $\mathbf{f}_{\mathbf{c}}\left(\mathbf{x}, \mathbf{x}_{\mathbf{r}}\right) \in \mathbb{R}^{n}$, where $\mathbf{x}_{\mathbf{r}}$ is the road surface configuration. The tire/road interaction is described in more detail in section 2.4. The external forces acting on the tire, e.g. a load 170 exerted on the hub of the tire, is denoted by $\mathbf{f}_{\mathbf{e}} \in \mathbb{R}^{n}$. This term is assumed to be constant. In this work, the hub forces are calculated by evaluating the components of the left-hand side terms of expression (1) corresponding with the tire-rim interface $\partial \Omega_{r}$. Summing over these force components in either the $x$ (longitudinal/rolling), $y$ (lateral/axial) and $z$ (vertical) directions then yields the corresponding hub force component for that direction.

The structural tire domain is denoted by $\Omega$, and is discretized by a set of finite elements $E$. The inner surface of the tire, upon which the air cavity pressure acts (the so-called inner liner), is denoted as the subdomain $\Omega_{p} \in \Omega$. Its boundary is denoted as $\partial \Omega_{p}$. This subdomain is discretized by the set of finite 180 elements $E_{p} \subset E$. The contact area of the tire tread is denoted by the subdomain $\Omega_{c} \in \Omega$. Its boundary is denoted as $\partial \Omega_{c}$. This subdomain is discretized by the set of finite elements $E_{c} \subset E$. The tire domain $\Omega$, tire-rim interface $\partial \Omega_{r}$ and subdomains $\Omega_{p}$ and $\Omega_{c}$, as well as their respective boundaries $\partial \Omega_{p}$ and $\partial \Omega_{c}$, are shown in Figure 1 . The acoustic air cavity domain is denoted by $\Omega_{A}$ and is shown in Figure 1 as well. A discretized tire domain $\Omega$ and acoustic domain $\Omega_{A}$ are shown in Figure 2. These meshes are used for the numerical validation in section 4 .

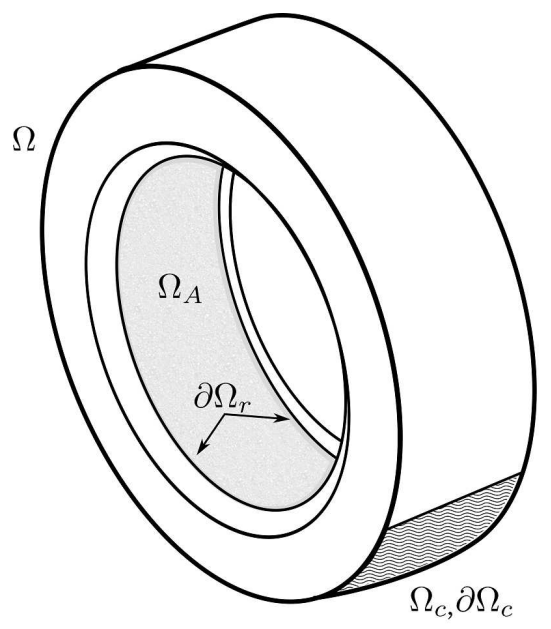

(a)

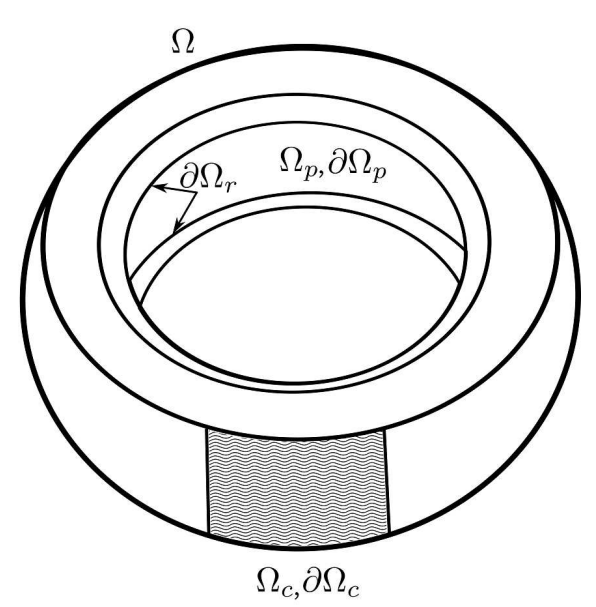

(b)

Figure 1: Example tire domain $\Omega$, the tire-rim interface $\partial \Omega_{r}$, the tire subdomains $\Omega_{p}$ and $\Omega_{c}$ and their respective boundaries $\partial \Omega_{p}$ and $\partial \Omega_{c}$, shown in both (a) and (b). The acoustic domain, i.e. the air cavity, is denoted as $\Omega_{A}$ and is shown in (a) 


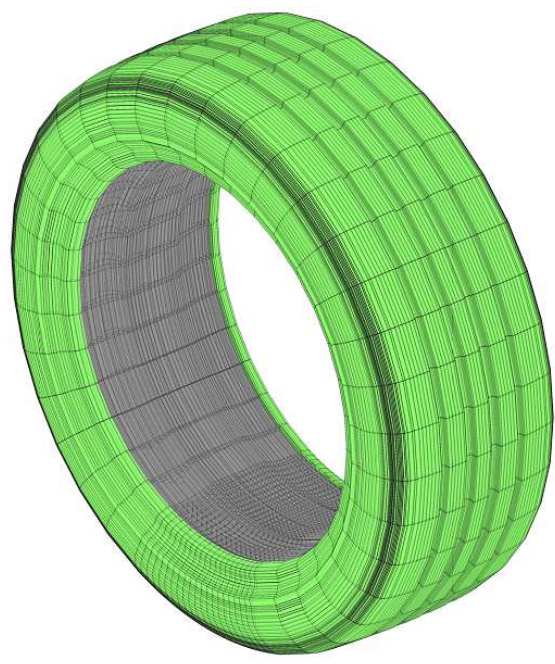

Figure 2: Actual discretized tire domain $\Omega$ as used for this work, shown in green, and a discretized acoustic domain $\Omega_{A}$, shown in gray

\subsection{The Arbitrary Lagrangian Eulerian formulation for bodies in rolling contact}

In this work, the ALE-formulation for bodies in rolling contact as proposed by Nackenhorst 22 is adopted to model the rolling of a tire with a constant angular velocity over a coarse road surface, as well as the associated dynamics. Conceptually a spatial mesh (which coincides with the tire domain $\Omega$ ) is used to describe the flow of tire material particles passing through spatial points (which are part of the spatial mesh) due to the rolling motion of the tire, rather than tracking the tire material particles individually. This corresponds to a Eulerian description of the tire material flow due to constant rolling. The deformation of the spatial mesh (resulting in the specific flow/streamlines of the tire material) itself is described using a Lagrangian formulation. This description of the tire deformation and dynamics corresponds to an observer describing the tire deformation and dynamics in a frame of reference that is fixed to the tire hub, but does not rotate along. Mathematically, the mapping of the reference configuration $\mathbf{x}_{\mathbf{0}}$ onto the current configuration $\mathbf{x}$ is split into a mapping onto an intermediate rigid body rotated reference configuration $\mathbf{x}_{\mathbf{0}}^{\mathbf{R}}$ and a mapping from this new reference configuration onto the current deformed configuration

$205 \mathbf{x}$. This idea is shown in Figure 3 . In comparison with a fully Lagrangian formulation, the ALE-formulation for bodies in rolling contact therefore replaces the reference configuration $\mathbf{x}_{\mathbf{0}}$ with the intermediate rigid body rotated reference configuration $\mathbf{x}_{\mathbf{0}}^{\mathbf{R}}$. The rigid body rotation (i.e. the mapping from $\mathbf{x}_{\mathbf{0}}$ to $\mathbf{x}_{\mathbf{0}}^{\mathbf{R}}$ ) itself is described using a Eulerian formulation, which results in a non-rotating 210 spatial mesh (i.e. not attached to material points of the tire domain). The deformation of the tire domain with respect to the new rigid body rotated reference configuration $\mathbf{x}_{\mathbf{0}}^{\mathbf{R}}$ (i.e. the mapping from $\mathbf{x}_{\mathbf{0}}^{\mathbf{R}}$ onto $\mathbf{x}$ ) is described using a Lagrangian formulation, i.e. using the displacements of material points. This 
mapping is thus described in the same way as in regular (Lagrangian) structural

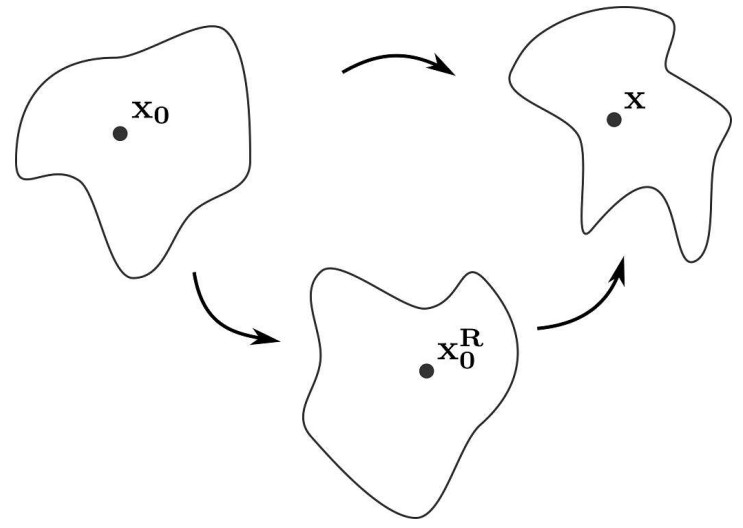

Figure 3: Mapping of the reference configuration $\mathbf{x}_{\mathbf{0}}$ onto a rigid body rotated configuration $\mathbf{x}_{\mathbf{0}}^{\mathbf{R}}$ and the current configuration $\mathbf{x}$. Figure adapted from Nackenhorst 22$]$

As a result, the deformation gradient tensor $\mathbf{F}$ of a material point is therefore split in a multiplicative way [22]:

$$
\mathbf{F}=\frac{\partial \mathbf{x}}{\partial \mathbf{x}_{\mathbf{0}}}=\frac{\partial \mathbf{x}}{\partial \mathbf{x}_{\mathbf{0}}^{\mathbf{R}}} \frac{\partial \mathbf{x}_{\mathbf{0}}^{\mathbf{R}}}{\partial \mathbf{x}_{\mathbf{0}}}=\left(\nabla_{\mathbf{R}} \mathbf{x}\right)\left(\nabla_{\mathbf{0}} \mathbf{x}_{\mathbf{0}}^{\mathbf{R}}\right)
$$

Here $\nabla$ is the so-called Del-operator ${ }^{\mathbb{1}}$, where $\nabla_{\mathbf{R}}$ is defined such that the partial derivatives are taken with respect to $\mathbf{x}_{\mathbf{0}}^{\mathbf{R}}$ and $\nabla_{\mathbf{0}}$ is defined such that the partial derivatives are taken with respect to $\mathbf{x}_{\mathbf{0}}$. The material time derivative is split additively into a relative and convective part:

$$
\frac{D}{D t}=\frac{\partial}{\partial t}+\left(\nabla_{\mathbf{R}} \mathbf{x}^{T}\right) \mathbf{w}
$$

Here $\mathbf{w} \in \mathbb{R}^{3}$ is the guiding velocity, defined as:

$$
\mathbf{w}=\mathbf{w}_{c}+\boldsymbol{\omega} \times \mathbf{x}_{r}
$$

Where $\mathbf{w}_{c}$ is the hub center velocity and $\mathbf{x}_{r}$ are the tire material point coordinates relative to the tire hub center. The tire angular velocity is denoted by $\boldsymbol{\omega} \in \mathbb{R}^{3}$. The weak form of the additional force terms that arise due to the use

${ }^{1} \nabla=\left(\frac{\partial}{\partial x_{1}}, \frac{\partial}{\partial x_{2}}, \frac{\partial}{\partial x_{3}}\right)^{T}$, where $x_{i}$ represents a Cartesian coordinate direction 
of ALE formulation is given by Nackenhorst [22] as:

$$
\begin{aligned}
\int_{\Omega} \rho\left[\delta \mathbf{x}^{T} \frac{\partial^{2} \mathbf{x}}{\partial t^{2}}+\delta \mathbf{x}^{T}\left(\left(\nabla_{\mathbf{R}} \frac{\partial \mathbf{x}^{T}}{\partial t}\right)\right.\right. & \mathbf{w})-\frac{\partial \mathbf{x}^{T}}{\partial t}\left(\left(\nabla_{\mathbf{R}} \delta \mathbf{x}^{T}\right) \mathbf{w}\right) \\
& \left.-\left(\left(\nabla_{\mathbf{R}} \delta \mathbf{x}^{T}\right) \mathbf{w}\right)^{T}\left(\left(\nabla_{\mathbf{R}} \mathbf{x}^{T}\right) \mathbf{w}\right)\right] d \Omega
\end{aligned}
$$

The flux terms as discussed by Nackenhorst 22] are neglected in this work, as a structured spatial discretization of the tire domain is used. More specifically, a symmetric discretization of the circumferential direction of the tire is used, effectively resulting in no net flux components [22]. Furthermore, $\rho$ is the material density of the particle located in the spatial location corresponding to the current configuration $\mathbf{x}$. Discretization of the additional ALE-terms (6) then yields the constant mass matrix $\mathbf{M} \in \mathbb{R}^{n \times n}$, the configuration dependent gyroscopic matrix $\mathbf{G}_{\mathbf{A L E}}(\mathbf{x}, \mathbf{w}) \in \mathbb{R}^{n \times n}$ and the configuration dependent gyroscopic force vector $\mathbf{f}_{\mathrm{ALE}}(\mathbf{x}, \mathbf{w}) \in \mathbb{R}^{n}$ as used in the equations of motion (1). The interested reader is referred to the works by Nackenhorst [13, 22] for more information regarding the definition and discretization of the ALE terms. Additionally, if pure steady-state rolling is considered (e.g. no excitation by road surface asperities), the time-dependent terms in the equations of motion (1) and expression (6) vanish [22]. This results in a purely static problem to be solved in order to obtain the steady-state (and static) rolling configuration:

$$
\mathbf{f}(\mathbf{x})-\mathbf{f}_{\mathrm{ALE}}(\mathbf{x}, \mathbf{w})=\mathbf{f}_{\mathbf{p}}\left(\mathbf{x}, p_{a}\right)+\mathbf{f}_{\mathbf{c}}\left(\mathbf{x}, \mathbf{x}_{\mathbf{r}}\right)+\mathbf{f}_{\mathbf{e}}
$$

Here, the contact force term is no longer time-dependent: the road surface constraint $\mathbf{x}_{\mathbf{r}}$ is constant. The steady-state rolling expression (7) is used to calculate the reduction basis, which is further explained in section 3.

\subsection{Nonlinear coupled vibro-acoustic tire model}

In order to include the vibro-acoustic coupling between the structural tire domain $\Omega$ and acoustic air cavity domain $\Omega_{A}$, the structural equations of motion (1) are extended using the linear structural displacement - acoustic pressure field formulation [30]:

$$
\begin{aligned}
{\left[\begin{array}{cc}
\mathbf{M} & \mathbf{0} \\
\mathbf{M}_{\mathrm{sa}} & \mathbf{M}_{\mathbf{a}}
\end{array}\right]\left[\begin{array}{c}
\ddot{\mathbf{x}} \\
\ddot{\mathbf{p}}
\end{array}\right]+\left[\begin{array}{cc}
\mathbf{G}_{\mathrm{ALE}} & \mathbf{0} \\
\mathbf{0} & \mathbf{0}
\end{array}\right]\left[\begin{array}{c}
\dot{\mathbf{x}} \\
\dot{\mathbf{p}}
\end{array}\right]+\left[\begin{array}{c}
\mathbf{K}_{\mathrm{sa}} \\
\mathbf{K}_{\mathbf{a}}
\end{array}\right]\left[\begin{array}{c}
\mathbf{u} \\
\mathbf{p}
\end{array}\right]+} \\
\\
{\left[\begin{array}{c}
\mathbf{f}-\mathbf{f}_{\mathrm{ALE}} \\
\mathbf{0}
\end{array}\right]=\left[\begin{array}{c}
\mathbf{f}_{\mathbf{p}}+\mathbf{f}_{\mathbf{c}}+\mathbf{f}_{\mathbf{e}} \\
\mathbf{0}
\end{array}\right] }
\end{aligned}
$$

Here $\mathbf{p} \in \mathbb{R}^{n_{a}}$ is the acoustic pressure field in $\Omega_{A}$, $\dot{\mathbf{p}}$ and $\ddot{\mathbf{p}}$ are its first and second derivatives with respect to time, respectively. Note that all of the dependencies of the structural domain force and matrix terms have been dropped for notation purposes. The acoustic mass matrix is denoted as $\mathbf{M}_{\mathbf{a}} \in \mathbb{R}^{n_{a} \times n_{a}}$ and the acoustic stiffness matrix is denoted as $\mathbf{K}_{\mathbf{a}} \in \mathbb{R}^{n_{a} \times n_{a}}$. As will be discussed in section 2.5. the acoustic damping arises from the numerical damping 
present in the time integrator. Therefore, no acoustic damping term is included explicitly. The vibro-acoustic coupling terms are denoted by $\mathbf{K}_{\text {sa }} \in \mathbb{R}^{n \times n_{a}}$ and $\mathbf{M}_{\mathbf{s a}} \in \mathbb{R}^{n_{a} \times n}$. Information on the weak form and discretization of these additional acoustic domain terms and vibro-acoustic coupling terms can be found in e.g. the work by Everstine [30. It is assumed that even though the displacements of the structural domain $\Omega$ at the structural-acoustic boundary can be large, the influence on the acoustic domain and vibro-acoustic coupling terms is limited. Therefore the general linear expressions are used. In the current work, the air is assumed to not rotate along with the tire; including rotation of the air would in fact require the use of a convected acoustic wave equation (as the air is no longer a quiescent medium), leading to additional terms and computational efforts [31, Chapter $9 \&$ Appendix F]. The presented approach can however be extended to include these effects.

\subsection{Tire/road rolling contact modeling}

240

The road surface asperities are one of the main sources of excitation for a tire rolling over a coarse road surface 32. The asperities come in and out of contact with the tire tread surface, impacting the tread and exciting the entire tire dynamically. A dynamic constraint-based contact mechanics approach, i.e. applying time-dependent geometrical contact constraints, is used in this work to model the tire/road surface interaction. Following the ALE-formulation idea of describing the flow of tire material in a Eulerian fashion, a similar idea is used to describe the contact constraints imposed by the road surface: the road material is also modeled as flowing through the spatial tire domain. This concept is shown in Figure 4 .

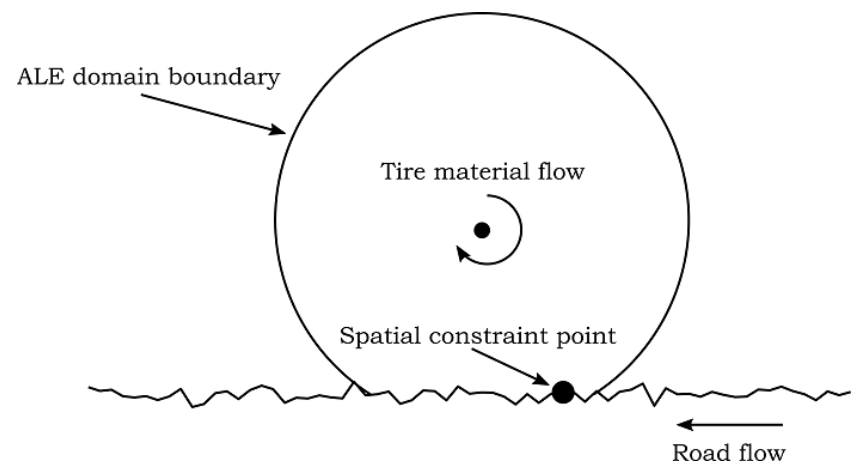

Figure 4: Schematic overview of applying dynamic contact constraints in the ALE-formulation

Due to the choice of the observer in the ALE-formulation (fixed to the tire hub), the road surface has a flow velocity equal in magnitude (opposite in sign) to the tire rolling speed. Using a Fourier series approximation, the road surface can thus be thought of as a combination of waves traveling in space with a velocity $v$. Therefore, as discussed by Brinkmeier et al. 11, given a frequency 
of interest $f$ and tire hub velocity $v$, the corresponding road surface wavelength $\lambda_{r}$ can be defined as:

$$
\lambda_{r}=\frac{v}{f}
$$

This wavelength $\lambda_{r}$ can be used as a spatial discretization criterion for the tire contact subdomain $\Omega_{c}$. Since geometrical constraints are imposed, a minimal amount of elements should be used per wavelength to correctly model the road surface geometry, and therefore meet the contact conditions [14. Depending on the coarseness of the road surface and the enveloping effect of the tire tread (where only parts of the tread and road surface are in contact), the amount of elements per wavelength $\lambda_{r}$ can be relaxed. A maximal element edge length $L_{e c}$ of an element in $E_{c}$ can therefore be expressed as:

$$
L_{e c}=\frac{v}{n_{\lambda_{r}} f}
$$

250 Where $n_{\lambda_{r}}$ is the amount of elements per wavelength and, as discussed before, depends on the road coarseness. Note that $n_{\lambda_{r}}$ can be determined by means of a convergence study, e.g. tracking the change in contact forces for different levels of tire subdomain $\Omega_{c}$ discretization. Given thus a road surface (i.e. a scan of a real road surface or an equivalent analytical expression of a road surface) and flow velocity $v$, the geometrical constraints acting on the tire contact subdomain $\Omega_{c}$ can be calculated a-priori per timestep. Example road surface scan segments, taken at different timesteps from the physical road surface used for the experimental and numerical validation testing in section 4 , is shown in Figure 5. Geometrical constraints extracted from this surface, corresponding to 260 a discrete point of the tire contact subdomain $\Omega_{c}$ are shown in Figure 6. The properties of the road surface and extracted constraints are discussed in more detail in section 4

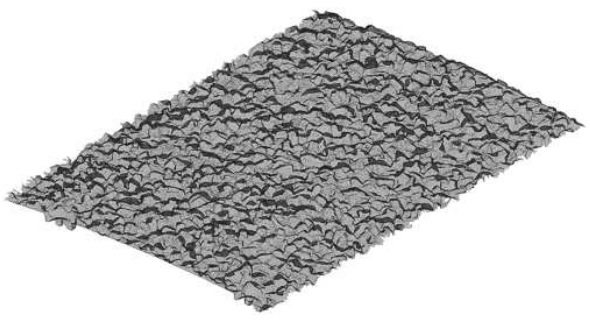

(a)

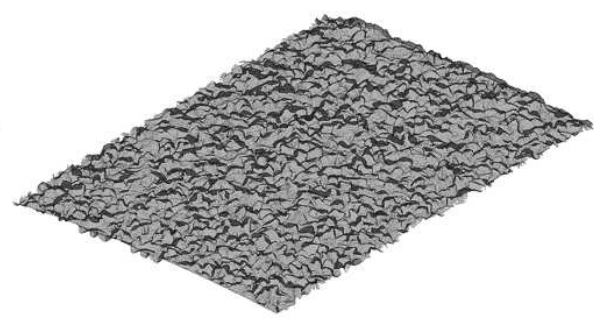

(b)

Figure 5: Example coarse road surface scan segments, corresponding to different timesteps 


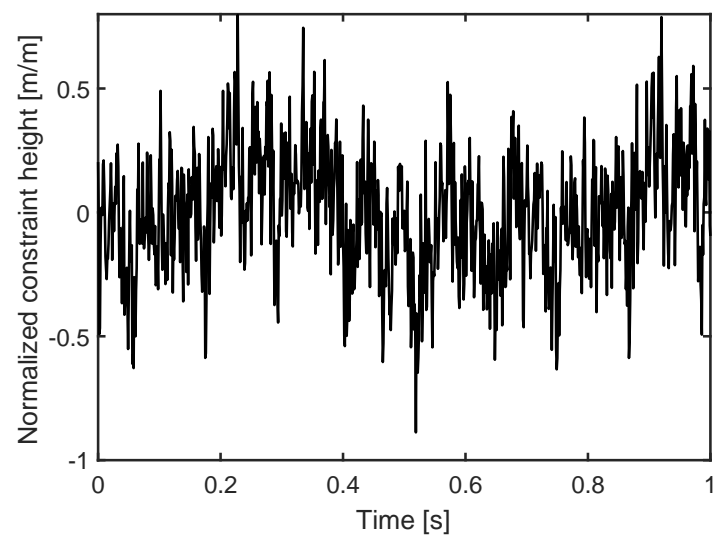

Figure 6: Example of geometrical contact constraints extracted in a spatial point of $\Omega_{c}$

Following Wriggers [14, Chapter 6], a classical penalty method approach is used to enforce the geometrical contact constraints. Assuming a constraint formulation for the normal contact and e.g. a Coulomb friction constitutive model for the tangential stick/slip contact, a general weak form expression for the contact force term $\mathbf{f}_{\mathbf{c}}$, using a weighted residuals approach (in analogy with e.g. an energy based approach [14, Chapter 6]), is given as:

$$
\int_{\partial \Omega_{c}} \delta \mathbf{g}_{n}^{T} \epsilon_{n} \mathbf{g}_{n}+\delta \mathbf{g}_{t}^{T} \epsilon_{t} \mathbf{g}_{t} d \partial \Omega_{c}
$$

Here $\partial \Omega_{c}$ again corresponds to the boundary of the contact interface part of the tire domain $\Omega_{c}, \mathbf{g}_{n}$ is the normal gap function, $\epsilon_{n}$ the normal contact penalty factor, $\mathbf{g}_{t}$ the tangential gap function and $\epsilon_{t}$ the tangential contact penalty factor (which can be expressed as a function of $\epsilon_{n}$ and the friction coefficient in the case of e.g. a regularized Coulomb frictional constitutive model). The normal gap function is defined such that the Kuhn-Karush-Tucker (KKT)/Hertz-SignoriniMoreau (HSM) conditions are met [14]. However, as the penalty method is used to enforce the contact constraints, the KKT/HSM conditions will be violated. Choosing a large enough penalty factor can minimize the violation of these conditions, but care must be taken such that the problem becomes not too numerically ill-conditioned [14, Chapter 6.3]. As free-rolling of the tire over the coarse road surface is considered in this work, frictionless contact is assumed, as the contribution of these friction forces to the structure borne interior noise is limited [32. As a result, the contact area is therefore assumed to be in a stick state (rather than a non free-rolling stick/slip state). The proposed approach can however be extended with frictional contact. The methodology for including frictional contact in the ALE-formulation can be found in the works by Nackenhorst [13, 22] and Ziefle and Nackenhorst [33]. Assuming frictionless contact, expression (11) becomes:

$$
\int_{\partial \Omega_{c}} \delta \mathbf{g}_{n}^{T} \epsilon_{n} \mathbf{g}_{n} d \partial \Omega_{c}
$$


As discussed before, advanced constitutive models could be used to further model the contact at smaller scales. The use of the constitutive model approach 265 does not impede with the approach further presented in this work, but typically requires, as mentioned before in section 1 , problem dependent constitutive parameters. In general this means the method would no longer be fully predictive.

The normal gap function $\mathbf{g}_{n} \in \mathbb{R}^{3}$ in a possible contact point $\bar{x}_{c}$ in the spatial tire contact domain $\Omega_{c}$ is defined as:

$$
\mathbf{g}_{n}=\max \left(\left(\left(\mathbf{x}_{\mathbf{r}}\left(\bar{x}_{c}\right)-\mathbf{x}\left(\bar{x}_{c}\right)\right)^{T} \mathbf{n}\left(\bar{x}_{c}\right)\right), 0\right) \mathbf{n}\left(\bar{x}_{c}\right)
$$

Here $\mathbf{x}_{\mathbf{r}}\left(\bar{x}_{c}\right)$ is the road surface configuration evaluated at $\bar{x}_{c}$, i.e. the geometrical road surface constraint active in $\bar{x}_{c}, \mathbf{x}\left(\bar{x}_{c}\right)$ is the tire configuration evaluated at $\bar{x}_{c}$ and $\mathbf{n}\left(\bar{x}_{c}\right)$ the unit outward normal on the road surface configuration in $\bar{x}_{c}$. For practical purposes, a mean unit outward normal is calculated for a set of road surface configurations corresponding to points in the vicinity of the possible contact point $\bar{x}_{c}$, i.e. within a user-defined radius of $\bar{x}_{c}$. In order to define this set of road surface configurations, a minimal distance or projection-based 275 contact detection algorithm as discussed by Wriggers [14, Chapter 10.1.2] can be used. Using the approach discussed earlier, the road surface configuration $\mathbf{x}_{\mathbf{r}}\left(\bar{x}_{c}\right)$ is calculated a-priori for all time instances $t$ of interest. Furthermore, the possible contact interface is known (due to the ALE-approach) and the road surface is assumed to be rigid. This last assumption is based on typical road material being much stiffer than the tire tread material. Therefore, the road surface can be modeled as a set of geometrical constraints active in the spatial tire contact domain. By considering the time-dependent road surface configurations in any $\bar{x}_{c} \in \Omega_{c}$, the corresponding time-dependent geometrical contact constraints active on $\Omega_{c}$ can be calculated a-priori and in parallel (as the road surface is 285 known per time $t$ ). This is a necessary condition in order for the suggested approach to be predictive. The well-known master-slave approach discussed in e.g. [14, Chapter 9] is used for the finite element implementation of [12). The rigid road surface is chosen to be the master body. As the unit normals do not change (due to the rigid body assumption), this further simplifies expres290 sion (12). The penalty factor $\epsilon_{n}$ is chosen iteratively [14, Chapter 10] such that a minimal penetration depth is achieved, without influencing the convergence behavior of the simulation.

As dynamic contact is considered, an additional contact condition needs to be taken into account. Apart from the KKT/HSM contact conditions, the socalled persistency condition [16, Chapter 7.2] needs to be included as well. This condition states that two points that are in contact need to have the same velocity as long as they are in contact (and thus be in persistent contact). For the tire/road contact problem, a tire material point is in persistent contact with a road surface asperity as long as said road asperity travels through the 300 tire footprint. Not enforcing the persistency condition results in tire material points coming into and out of contact with the road surface, during the travel of the road asperities through the tire footprint. As discussed by Suwannachit et al. 34, using an adapted time integrator (as proposed by e.g. Laursen [16, 
Chapter 7]) or having enough numerical dissipation in the integrator leads to enforcement of the persistency constraint. Therefore, in this work the generalized$\alpha$ (the time integrator, discussed in section 2.5 $\rho_{\infty}$ parameter is set to a low value to ensure the persistency condition. Furthermore, the set of contact constraints is applied and held constant for multiple subsequent timesteps, in order to ensure that the persistency condition can be met. By applying the same set of 310 contact constraints for multiple subsequent timesteps, impact-effects are allowed to decay: contacting nodes are allowed to converge within a small time-window to a persistent contact state. The timestep therefore has to be chosen such that the persistency condition can be met and the relevant system dynamics can be captured.

\subsection{Time integration}

A variant of the implicit generalized- $\alpha$ discrete time integration method, proposed by Arnold and Brüls [35], is chosen to time-discretize the equations of motion (1). This variant of the generalized- $\alpha$ method has second-order accuracy for the structural acceleration field variables. Theory and implementation details can be found in 35. An implicit rather than an explicit time integration scheme is chosen, as this allows to use larger timesteps $\Delta t$ while still maintaining stability. This results in less timesteps to be evaluated. As an iterative implicit scheme is used, the nonlinear equations of motion (8) are consistently linearized [27, Chapter 8.4] in the spatial domain around the current configuration $\mathbf{x}$. This yields the following set of equations:

$$
\begin{aligned}
{\left[\begin{array}{cc}
\mathbf{M} & \mathbf{0} \\
\mathbf{M}_{\mathbf{s a}} & \mathbf{M}_{\mathbf{a}}
\end{array}\right]\left[\begin{array}{c}
\ddot{\mathbf{x}} \\
\ddot{\mathbf{p}}
\end{array}\right]+\left[\begin{array}{cc}
\mathbf{G}_{\mathrm{ALE}} & \mathbf{0} \\
\mathbf{0} & \mathbf{0}
\end{array}\right]\left[\begin{array}{c}
\dot{\mathbf{x}} \\
\dot{\mathbf{p}}
\end{array}\right]+\left[\begin{array}{c}
\mathbf{K}_{\mathbf{s a}} \\
\mathbf{K}_{\mathbf{a}}
\end{array}\right]\left[\begin{array}{c}
\mathbf{u} \\
\mathbf{p}
\end{array}\right]+} \\
{\left[\mathbf{K}-\mathbf{K}_{\mathbf{p}}-\mathbf{K}_{\mathbf{c}}-\mathbf{K}_{\mathbf{A L E}}\right]\left[\begin{array}{c}
\Delta \mathbf{u} \\
\mathbf{0}
\end{array}\right]=\left[\begin{array}{c}
\mathbf{f}_{\mathbf{p}}+\mathbf{f}_{\mathbf{c}}+\mathbf{f}_{\mathbf{e}}-\left(\mathbf{f}-\mathbf{f}_{\mathrm{ALE}}\right) \\
\mathbf{0}
\end{array}\right] }
\end{aligned}
$$

Here $\mathbf{K} \in \mathbb{R}^{n \times n}$ is the internal force tangent stiffness matrix, $\mathbf{K}_{\mathbf{p}} \in \mathbb{R}^{n \times n}$ is the pressure load force tangent stiffness matrix, $\mathbf{K}_{\mathbf{c}} \in \mathbb{R}^{n \times n}$ is the contact force tangent stiffness matrix and $\mathbf{K}_{\mathrm{ALE}} \in \mathbb{R}^{n \times n}$ is the ALE inertia matrix [22. Typically the numerical costs of one iteration step of an implicit time integrator exceed those of an explicit time integrator, due to the additional tangential stiffness terms that need to be evaluated and the large linear system that needs to be solved. Therefore, the benefit of having fewer timesteps is typically lost, as the total computational time of one implicit iteration step is typically equal to the total computational time of multiple explicit iteration steps. In the proposed fully predictive high-fidelity approach however, only a limited set of elements needs to be evaluated (because of the hyper-reduction) and a small linear system has to be solved per iteration step. As the additional computational time per implicit hyper-reduced iteration step is very limited with respect to an explicit hyper-reduced iteration step, the use of the hyper-reduction method 330 therefore enables the use of an implicit time integrator and, as a result, the use of larger timesteps. Details of the hyper-reduction method are given in section 3. The numerical damping of the generalized- $\alpha$ method can be controlled 
by the $\rho_{\infty}$ parameter: $\rho_{\infty}=1$ results in no damping, whereas $\rho_{\infty}=0$ leads to so-called annihilation of the high-frequency response [35. Therefore, $\rho_{\infty}$ is set close to 1 for the acoustic domain (i.e. low numerical damping) and close to 0 for the structural domain (to meet the persistency condition, as discussed in section 2.4 .

\section{Reduced order tire modeling}

Solving the set of linearized equations of motions (14) per iteration step quickly becomes very costly as the amount of degrees of freedom increases. For industrial-sized design problems, e.g. the tire/road contact problem discussed in this work, the total computational cost becomes too large and the simulations are no longer feasible to use in a design context. Therefore, a nonlinear MOR technique is applied to expression (14).

\subsection{Reduced order basis definition for tire modeling}

The full-order solution $\left[\begin{array}{ll}\mathbf{u} & \mathbf{p}\end{array}\right]^{T} \in \mathbb{R}^{n+n_{a}}$, residing in the solution manifold defined by 8 , is approximated by the reduced-order solution $\left[\begin{array}{ll}\mathbf{q}_{\mathbf{s}} & \mathbf{q}_{\mathbf{a}}\end{array}\right]^{T} \in \mathbb{R}^{m}$, which resides in a constant subspace of the solution manifold. The subspace is spanned by the columns of $\mathbf{V} \in \mathbb{R}^{n+n_{a} \times m}$, where:

$$
\mathbf{V}=\left[\begin{array}{l}
\mathbf{V}_{\mathbf{s}} \\
\mathbf{V}_{\mathbf{a}}
\end{array}\right]
$$

Here $\mathbf{V}_{\mathbf{s}} \in \mathbb{R}^{n \times m}$ is defined as the structural part of the subspace basis and $\mathbf{V}_{\mathbf{a}} \in$ $\mathbb{R}^{n_{a} \times m}$ is defined as the acoustic part of the subspace basis. Hereafter $\mathbf{V}$ is called the reduced order basis (ROB). This leads to the following approximations:

$$
\begin{aligned}
& \mathbf{x} \approx \tilde{\mathbf{x}}=\mathrm{x}_{\mathbf{0}}+\mathrm{V}_{\mathrm{s}} \mathbf{q}_{\mathrm{s}} \\
& \mathbf{p} \approx \tilde{\mathbf{p}}=\mathrm{V}_{\mathrm{a}} \mathbf{q}_{\mathrm{a}}
\end{aligned}
$$

And since $\mathbf{V}$ is chosen to be constant, it follows that:

$$
\begin{aligned}
& \dot{\mathbf{x}} \approx \dot{\tilde{\mathbf{x}}}=\mathbf{V}_{\mathbf{s}} \dot{q}_{\mathrm{s}} \\
& \dot{\mathbf{p}} \approx \dot{\tilde{\mathbf{p}}}=\mathbf{V}_{\mathbf{a}} \dot{q}_{\mathbf{a}} \\
& \ddot{\mathbf{x}} \approx \ddot{\tilde{\mathbf{x}}}=\mathbf{V}_{\mathbf{s}} \ddot{\mathbf{q}}_{\mathrm{s}} \\
& \ddot{\mathbf{p}} \approx \ddot{\tilde{\mathbf{p}}}=\mathbf{V}_{\mathrm{a}} \ddot{\dot{q}_{\mathrm{a}}}
\end{aligned}
$$

\section{2. (Hyper-)Reduced equations of motion}

When inserting the approximations $(16)-\sqrt{21})$ in the linearized equations of motion (14), this introduces an error term which can be eliminated by the orthogonal projection (i.e. premultiplication with $\mathbf{V}^{T}$ ) of the linearized equations of motion (14) onto the ROB V. This is a direct result of the application of the Galerkin method, where the test functions are chosen to be same as the solution 
functions, yielding an error term orthogonal to the test function space which is thus effectively eliminated after the premultiplication by $\mathbf{V}^{T}$. It should thus be noted that this eliminated error term does not arise due to the difference between the full-order solutions and the approximations (16) $-(21)$, but due to the application of the Galerkin method. This then results in the ROM:

$$
\tilde{\mathbf{M}}\left[\begin{array}{c}
\ddot{q}_{s} \\
\ddot{q}_{a}
\end{array}\right]+\tilde{\mathbf{G}}\left[\begin{array}{c}
\dot{q}_{s} \\
\dot{q}_{a}
\end{array}\right]+\tilde{\mathbf{K}}\left[\begin{array}{c}
\Delta \mathbf{q}_{s} \\
0
\end{array}\right]+\tilde{\mathbf{K}}_{\mathrm{sa}}\left[\begin{array}{l}
\mathbf{q}_{s} \\
\mathbf{q}_{\mathrm{a}}
\end{array}\right]=\tilde{\mathbf{f}}
$$

Where

$$
\begin{aligned}
\tilde{\mathbf{M}} & =\left[\begin{array}{cc}
\mathbf{V}_{\mathbf{s}}^{T} \mathbf{M V}_{\mathbf{s}} & \mathbf{0} \\
\mathbf{V}_{\mathbf{a}}^{T} \mathbf{M}_{\mathbf{s a}} \mathbf{V}_{\mathbf{s}} & \mathbf{V}_{\mathbf{a}}^{T} \mathbf{M}_{\mathbf{a}} \mathbf{V}_{\mathbf{a}}
\end{array}\right] \in \mathbb{R}^{m \times m} \\
\tilde{\mathbf{G}} & =\left[\begin{array}{cc}
\mathbf{V}_{\mathbf{s}}^{T} \mathbf{G}_{\mathbf{A L E}} \mathbf{V}_{\mathbf{s}} & \mathbf{0} \\
\mathbf{0} & \mathbf{0}
\end{array}\right] \in \mathbb{R}^{m \times m} \\
\tilde{\mathbf{K}} & =\left[\begin{array}{cc}
\mathbf{V}_{\mathbf{s}}^{T}\left(\mathbf{K}-\mathbf{K}_{\mathbf{p}}-\mathbf{K}_{\mathbf{c}}-\mathbf{K}_{\mathbf{A L E}}\right) \mathbf{V}_{\mathbf{s}} & \mathbf{0} \\
\mathbf{0} & \mathbf{0}
\end{array}\right] \in \mathbb{R}^{m \times m} \\
\tilde{\mathbf{K}}_{\mathbf{s a}} & =\left[\begin{array}{l}
\mathbf{V}_{\mathbf{s}}^{T} \mathbf{K}_{\mathbf{s a}} \mathbf{V}_{\mathbf{a}} \\
\mathbf{V}_{\mathbf{a}}^{T} \mathbf{K}_{\mathbf{a}} \mathbf{V}_{\mathbf{a}}
\end{array}\right] \in \mathbb{R}^{m \times m} \\
\tilde{\mathbf{f}} & =\left[\begin{array}{c}
\mathbf{V}_{\mathbf{s}}^{T}\left(\mathbf{f}_{\mathbf{p}}+\mathbf{f}_{\mathbf{c}}+\mathbf{f}_{\mathbf{e}}-\mathbf{f}\right) \\
\mathbf{0}
\end{array}\right] \in \mathbb{R}^{m}
\end{aligned}
$$

Here the projection onto the structural part of the ROB, $\mathbf{V}_{\mathbf{s}}$, and acoustic part of the ROB, $\mathbf{V}_{\mathbf{a}}$, has been split in order preserve stability [36]. The reduced mass matrix $\tilde{\mathbf{M}}$, reduced external force $\mathbf{V}_{\mathbf{s}}{ }^{T} \mathbf{f}_{\mathbf{e}}$ and the reduced vibro-acoustic coupling term $\tilde{\mathbf{K}}_{\mathbf{s a}}$ can be calculated a-priori, as they are configuration independent and remain constant. The other reduced terms are configuration-dependent and therefore have to be evaluated again per iteration step. A second-tier hyperreduction approximation of these terms is used to reduce the computational cost associated with their evaluation [26].

The ROB as used in this work is generated using nonlinear steady-state (which are in fact static due to the ALE formulation 22]) contact configurations $\chi \in \mathbb{R}^{n \times n_{\chi}}$ and vibro-acoustic eigenmodes $\boldsymbol{\Psi} \in \mathbb{R}^{n+n_{a} \times n_{\Psi}}$, where $n_{\chi}+n_{\Psi}=m$ and:

$$
\Psi=\left[\begin{array}{l}
\Psi_{\mathrm{s}} \\
\boldsymbol{\Psi}_{\mathrm{a}}
\end{array}\right]
$$

Here $\boldsymbol{\Psi}_{\mathbf{s}} \in \mathbb{R}^{n \times n_{\Psi}}$ and $\boldsymbol{\Psi}_{\mathrm{a}} \in \mathbb{R}^{n_{a} \times n_{\Psi}}$. The structural and acoustic part of the ROB are then defined as:

$$
\begin{aligned}
\mathbf{V}_{\mathbf{s}} & =\left[\begin{array}{lllllll}
\chi^{1}-\mathbf{x}_{\mathbf{0}} & \cdots & \chi^{n_{\chi}}-\mathbf{x}_{\mathbf{0}} & \mathbf{\Psi}_{\mathbf{s}}{ }^{1} & \cdots & \mathbf{\Psi}_{\mathbf{s}}{ }^{n_{\Psi}}
\end{array}\right] \in \mathbb{R}^{n \times m} \\
\mathbf{V}_{\mathbf{a}} & =\left[\begin{array}{lllll}
\mathbf{0} & \mid & \mathbf{\Psi}_{\mathbf{a}}{ }^{1} & \cdots & \boldsymbol{\Psi}_{\mathbf{a}}{ }^{n_{\Psi}}
\end{array}\right] \in \mathbb{R}^{n_{a} \times m}
\end{aligned}
$$

This ROB can be calculated a-priori and in parallel. A set of road surface constraints $\left[\begin{array}{lll}\mathbf{x}_{\mathbf{r}}{ }^{1} & \cdots & \mathbf{x}_{\mathbf{r}}{ }^{n_{\chi}}\end{array}\right]$ is sampled equidistantly from the set of a-priori 
known road surface constraints. The corresponding steady-state (static) contact configurations $\chi^{k}, k=1 \cdots n_{\chi}$ are calculated as follows, using expression (7):

$$
\mathbf{f}\left(\chi^{k}\right)-\mathbf{f}_{\mathrm{ALE}}\left(\chi^{k}\right)=\mathbf{f}_{\mathbf{p}}\left(\chi^{k}, p_{a}\right)+\mathbf{f}_{\mathbf{c}}\left(\chi^{k}, \mathbf{x}_{\mathbf{r}}{ }^{k}\right)+\mathbf{f}_{\mathbf{e}}
$$

These contact configurations can be considered as nonlinear constraint modes, where a distributed set of constraints (the geometrical contact constraints) is applied rather than local unit displacements (as is the case for linear constraint modes [37). An example set of nonlinear constraint modes is shown in Figure 8 . The amount of nonlinear constraint modes depends on the type of road surface and total amount of rolling distance/time that is simulated. A convergence criterion based on the static projection error can be used to select the minimum amount of nonlinear constraint modes. Given a nonlinear constraint mode $\chi^{*}$ corresponding to a road surface constraint $\mathbf{x}_{\mathbf{r}}{ }^{*} \notin\left[\begin{array}{lll}\mathbf{x}_{\mathbf{r}}{ }^{1} & \cdots & \mathbf{x}_{\mathbf{r}}{ }^{n_{\chi}}\end{array}\right]$, the static projection error $\epsilon_{\chi}$ for a static structural reduction basis $\mathbf{V}_{\chi}=$ $\left[\begin{array}{lll}\chi^{1}-\mathbf{x}_{\mathbf{0}} & \cdots & \chi^{n_{\chi}}-\mathbf{x}_{\mathbf{0}}\end{array}\right]$ can be defined as:

$$
\epsilon_{\chi}=\frac{\left\|\left(\chi^{*}-\mathbf{x}_{\mathbf{0}}\right)-\mathbf{V}_{\chi}\left(\mathbf{V}_{\chi}^{T} \mathbf{V}_{\chi}\right)^{-1} \mathbf{V}_{\chi}^{T}\left(\chi^{*}-\mathbf{x}_{\mathbf{0}}\right)\right\|_{2}}{\left\|\chi^{*}-\mathbf{x}_{\mathbf{0}}\right\|_{2}}
$$

355 Here $\|\cdot\|_{2}$ denotes the $L_{2}$-norm. Given then a first choice of $n_{\chi}$ and a userdefined tolerance $\tau_{\chi}$, the number of nonlinear constraint modes can be selected using e.g. Algorithm 1. If the convergence criterion is not met for a given $n_{\chi}$, the tolerance $\tau_{\chi}$ can either be increased or additional nonlinear constraint modes can be calculated (i.e. increasing $n_{\chi}$ ). The convergence behavior of $\epsilon_{\chi}$ it is clear that a minimal number of nonlinear constraint modes can be selected for which the static projection error converges.

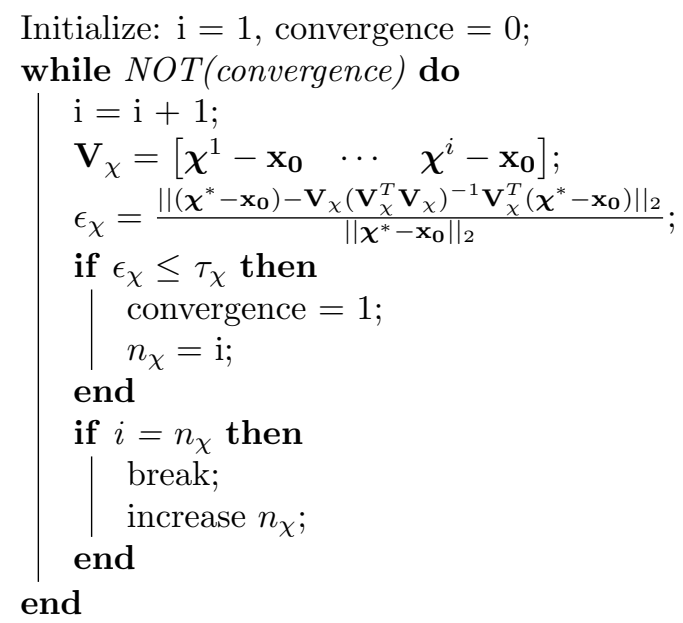

Algorithm 1: Selecting the number of nonlinear constraint modes 


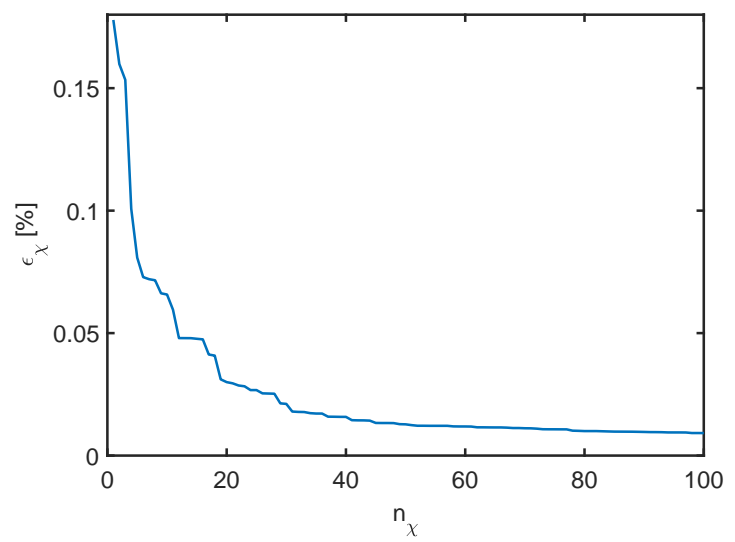

Figure 7: Convergence behavior of $\epsilon_{\chi}$ for different values of $n_{\chi}$

Following the MEM method [23], sets of eigenmodes are calculated around nonlinear constraint modes $\chi^{l} \in \chi, l=1 \cdots n_{l} \leq n_{\chi}$ :

$$
\boldsymbol{\Psi}_{\boldsymbol{\chi}^{l}}=\operatorname{eig}\left(\overline{\overline{\mathbf{K}}}\left(\chi^{l}\right), \overline{\overline{\mathbf{M}}}\right) \in \mathbb{R}^{n+n_{a} \times n_{\Psi}}
$$

Here

$$
\overline{\bar{M}}=\left[\begin{array}{cc}
\mathbf{M} & 0 \\
\mathbf{M}_{\mathbf{s a}} & \mathbf{M}_{\mathbf{a}}
\end{array}\right]
$$

and

$$
\overline{\overline{\mathbf{K}}}=\left[\begin{array}{cc}
\mathbf{K}-\mathbf{K}_{\mathbf{p}}-\mathbf{K}_{\mathbf{c}}-\mathbf{K}_{\mathrm{ALE}} & \mathbf{K}_{\mathbf{s a}} \\
\mathbf{0} & \mathbf{K}_{\mathbf{a}}
\end{array}\right]
$$

Each set of eigenmodes should at least span the frequency range of interest in order to capture the relevant dynamics. The sets of eigenmodes are then concatenated:

$$
\Psi_{\chi}=\left[\begin{array}{lll}
\Psi_{\chi^{1}} & \cdots & \Psi_{\chi^{n_{l}}}
\end{array}\right]
$$

And a singular value decomposition is performed on the concatenated eigenspace, where the $n_{\Psi}$ most dominant contributions are kept:

$$
\begin{aligned}
\mathbf{U} \boldsymbol{\Sigma} \mathbf{W}^{T} & =\operatorname{svd}\left(\Psi_{\chi}\right) \\
\Psi & =\mathbf{U}_{i, i=1 \cdots n_{\Psi}}
\end{aligned}
$$

An example set of eigenmodes is shown in Figure 8 as well. 


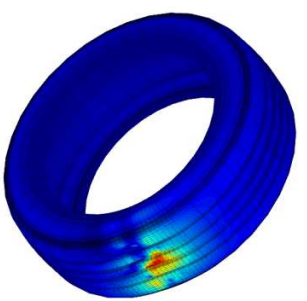

(a)

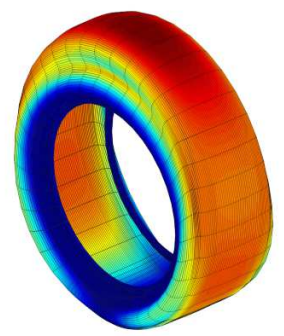

(d)

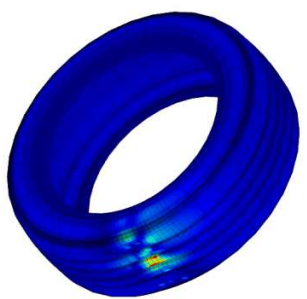

(b)

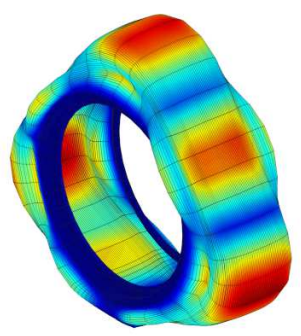

(e)

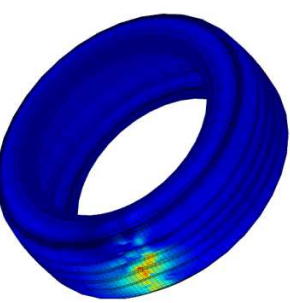

(c)

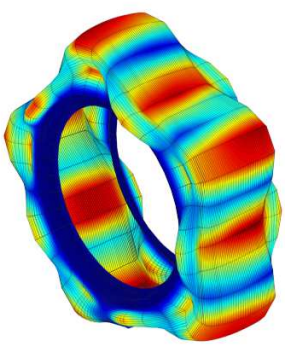

(f)

Figure 8: Nonlinear static contact configurations (a)-(c) and eigenmodes (d)-(f) as used for the ROB. Deformations have been scaled for illustration purposes

As discussed before, a second-tier approximation of the reduced configuration dependent terms is performed. Following the finite element assembly procedure, the reduced configuration dependent terms in 22 can be written as:

$$
\tilde{\mathbf{f}}(\mathbf{x})=\mathbf{V}_{\mathbf{s}}^{T} \mathbf{f}(\mathbf{x})=\sum_{i=1}^{|E|} \mathbf{V}_{\mathbf{s}, i}^{T} \mathbf{f}_{i}(\mathbf{x})
$$

Here $E$ is the total set of elements and $\mathbf{V}_{\mathbf{s}, i}^{T}$ and $\mathbf{f}_{i}$ have a sparse structure, only containing the contributions of element $i$. These reduced terms can be approximated further by using the Energy-Conserving Sampling and Weighting (ECSW) hyper-reduction approach as proposed by Farhat et al. [26]:

$$
\tilde{\mathbf{f}}(\mathbf{x}) \approx \overline{\mathbf{f}}(\mathbf{x})=\sum_{i=1}^{\left|E^{s}\right|} s_{i} \mathbf{V}_{\mathbf{s}, i}^{T} \mathbf{f}_{i}(\mathbf{x}) \quad \text { and } \quad s_{i} \geq 0
$$

Here the reduced set of finite elements $E^{s} \subset E$ and their respective weights $s_{i}$ are calculated (sampled from the full set $E$ ) using dynamic time-domain training data. Given a set of training samples $\mathbf{x}_{i}, i=1 \cdots n_{s}$, the reduced set of elements $E^{s}$ and their respective nonnegative weights $\mathbf{s}$ are calculated in order to match the hyper-reduced and reduced internal forces. Since only a reduced set of elements, $E^{s}$, needs to be evaluated per iteration step, large computational speedups can be achieved. This second-tier hyper-reduction step is necessary to come up with a computational efficient model. Typically, multiple dynamic 
time-domain simulations need to be run to generate the required training samples. As the total simulation cost for the off-line training phase quickly grows too large (since multiple simulations need to be run per tire design/tire design change), application of the ECSW method in the proposed fully predictive high-fidelity approach is not feasible. Therefore in this work the a-priori MultiExpansion Modal Reduction method (MEM) [23, 24, 25] is used to calculate the reduced set of elements $E^{s}$ and their respective nonnegative weights $\mathbf{s}$. Here static configurations, corresponding to the nonlinear constraint modes $\chi^{k}$, are used rather than time-domain training data. The required amount of samples to perform the element sampling for the tire/road case is discussed in more detail in 25. As discussed before, the nonlinear constraint modes can be calculated in parallel and a-priori, therefore making the application of the second-tier hyperreduction feasible. Application of the MEM method to the reduced equations of motion 22 then leads to:

$$
\tilde{\mathbf{M}}\left[\begin{array}{c}
\ddot{q}_{s} \\
\ddot{q}_{a}
\end{array}\right]+\overline{\mathbf{G}}\left[\begin{array}{c}
\dot{q}_{s} \\
\dot{q}_{a}
\end{array}\right]+\overline{\mathbf{K}}\left[\begin{array}{c}
\Delta \mathbf{q}_{s} \\
\mathbf{0}
\end{array}\right]+\tilde{\mathbf{K}}_{\mathrm{sa}}\left[\begin{array}{l}
\mathbf{q}_{s} \\
\mathbf{q}_{\mathrm{a}}
\end{array}\right]=\overline{\mathbf{f}}
$$

Here the reduced and hyper-reduced terms are:

$$
\begin{aligned}
\overline{\mathbf{G}} & =\left[\begin{array}{cc}
\sum_{i=1}^{\left|E^{s}\right|} s_{i} \mathbf{V}_{\mathbf{s}, i}^{T} \mathbf{G}_{i, \mathbf{A L E}} \mathbf{V}_{\mathbf{s}, i} & \mathbf{0} \\
\mathbf{0} & \mathbf{0}
\end{array}\right] \\
\overline{\mathbf{K}} & =\left[\begin{array}{cc}
\sum_{i=1}^{\left|E^{s}\right|} s_{i} \mathbf{V}_{\mathbf{s}, i}^{T}\left(\mathbf{K}_{i}-\mathbf{K}_{i, \mathbf{A L E}}\right) \mathbf{V}_{\mathbf{s}, i}-\sum_{i=1}^{\left|E_{p}^{s}\right|} s_{i, \mathbf{p}} \mathbf{V}_{\mathbf{s}, i}^{T} \mathbf{K}_{i, \mathbf{p}} \mathbf{V}_{\mathbf{s}, i}-\mathbf{V}_{\mathbf{s}}{ }^{T} \mathbf{K}_{\mathbf{c}} \mathbf{V}_{\mathbf{s}} & \mathbf{0} \\
\mathbf{0} & \mathbf{0}
\end{array}\right] \\
\overline{\mathbf{f}} & =\left[\begin{array}{c}
\left.\sum_{i=1}^{\left|E_{p}^{s}\right|} s_{i, \mathbf{p}} \mathbf{V}_{\mathbf{s}, i}^{T} \mathbf{f}_{i, \mathbf{p}}+\mathbf{V}_{\mathbf{s}}^{T} \mathbf{f}_{\mathbf{c}}+\mathbf{V}_{\mathbf{s}}^{T} \mathbf{f}_{\mathbf{e}}-\sum_{i=1}^{\left|E^{s}\right|} s_{i} \mathbf{V}_{\mathbf{s}, i}^{T}\left(\mathbf{f}_{i}-\mathbf{f}_{i, \mathbf{A L E}}\right)\right] \\
\mathbf{0}
\end{array}\right]
\end{aligned}
$$

While all of the elements $E$ are used to perform the MEM sampling of the in-

ternal and ALE force terms and to assemble the hyper-reduced form

$\sum_{i=1}^{\left|E^{s}\right|} s_{i} \mathbf{V}_{\mathbf{s}, i}^{T}\left(\mathbf{f}_{i}-\mathbf{f}_{i, \mathbf{A L E}}\right)$, the subset of elements, $E_{p} \subset E$ used to discretize $\Omega_{p}$, is used to perform the MEM sampling of the pressure force term and assemble the hyper-reduced form $\sum_{i=1}^{\left|E_{p}^{s}\right|} s_{i, \mathbf{p}} \mathbf{V}_{\mathbf{s}, i}^{T} \mathbf{f}_{i, \mathbf{p}}$. Due to the use of the ALEformulation, the tire/road contact area is known a-priori and the geometric contact constraints are calculated a-priori as well. Therefore, no contact search has to be performed per iteration step. The contact force term $\mathbf{V}_{\mathbf{s}}{ }^{T} \mathbf{f}_{\mathbf{c}}$ is therefore projected per iteration step, as hyper-reduction does not offer any benefit in the specific tire/road application. Recently, the use and computational benefits of hyper-reduction for contact problems has been shown by Blockmans [38]. An 375 example of a reduced set of elements is shown in Figure 9 . 


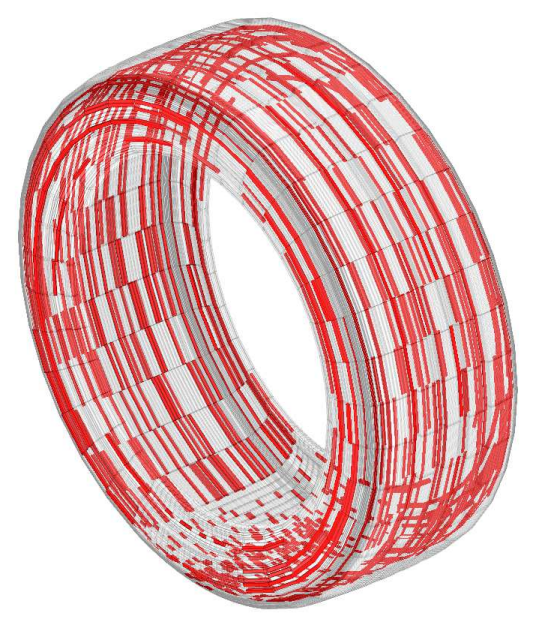

Figure 9: Example of a reduced set of elements $E^{s}$, sampled from the element set $E$ used to discretize the tire domain $\Omega$

\section{Experimental reduced-order tire model validation: Hub force eval- uation}

The proposed predictive method is validated by means of experimental vertical hub force measurements. The experimental and numerical test setup, as well as the numerical simulation setup, are discussed below. A comparison between experimental data and numerical simulation data is made as well. As already discussed in section 1. two alternative linearized approaches are proposed and compared with the reduced fully nonlinear $\mathrm{FE}$ approach to demonstrate the considerably improved accuracy through accounting for the nonlinear tire behavior.

\subsection{Experimental test setup}

A test tire of size 205/55R 16 is loaded force-controlled, using a vertical external force $\left\|\mathbf{f}_{\mathrm{e}}\right\|_{2}=4000 \mathrm{~N}$, onto a drum. After achieving the desired static vertical load, the axle height is locked and the drum is set into motion. The drum rotates with a fixed angular velocity and drives the tire. The tire can thus be seen as rolling in free-rolling condition. It should be noted that as a result of the rotation, the mean dynamic vertical load will be larger than the static vertical load. The drum angular velocity is chosen such that the rolling speed of the tire is $22.22 \mathrm{~ms}^{-1}$, i.e. $80 \mathrm{kmh}^{-1}$. A coarse road surface is mounted on the drum. The 395 maximum spatial wavelength of the coarse road surface is equal to $9.61 \mathrm{~m}$ for one drum rotation, with a Mean Profile Depth (MPD) of $3.4 \cdot 10^{-3} \mathrm{~m}$. Furthermore, the nominal stone chip size ranges between $4 \cdot 10^{-3} \mathrm{~m}$ and $15 \cdot 10^{-3} \mathrm{~m}$. A surface scan of this mounted road surface (i.e. taking into account the drum dimensions as well) is used for the numerical simulations, where a $10^{-4} \mathrm{~m}$ sampling length is used to generate the surface scan. Using Nyquist's theorem and expression (9), 
this corresponds to a minimum excitation frequency of $2.3 \mathrm{~Hz}$ and a maximum excitation frequency of $110 \mathrm{kHz}$ for the tire rolling speed of $22.22 \mathrm{~ms}^{-1}$ for one drum rotation. The normalized surface height distribution of a segment of the surface scan is shown in Figure 10.

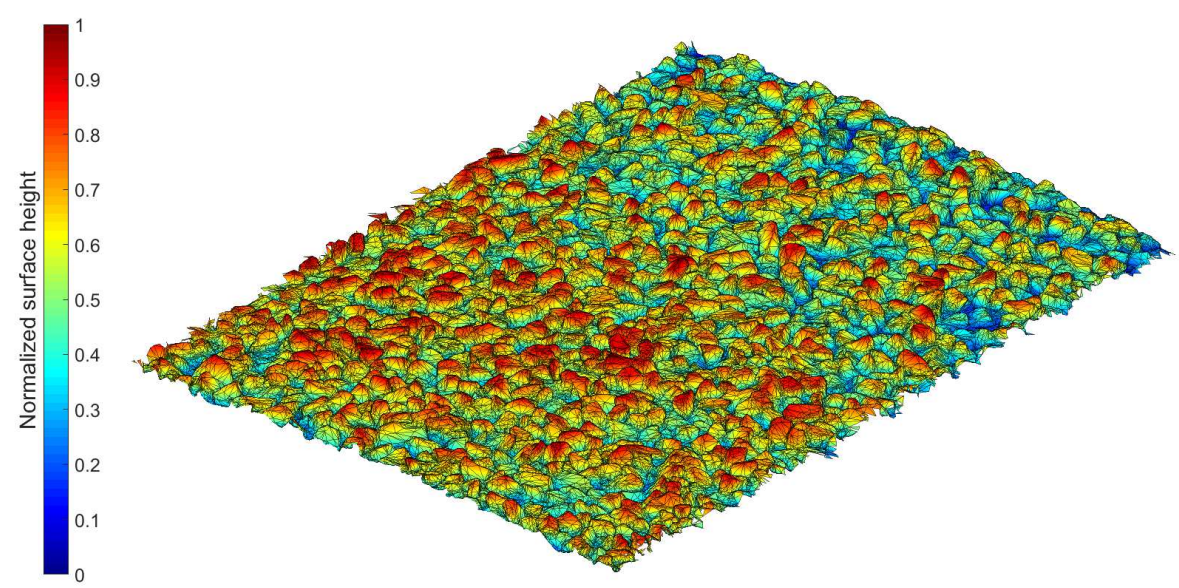

Figure 10: Road surface height distribution for a segment of the surface scan

\subsection{Numerical simulation setup}

A fully nonlinear vibro-acoustic finite element model of the test tire is constructed using geometrical properties extracted from the test tire design. As $400 \mathrm{~Hz}$ is the maximum excitation frequency of interest in this work, expression $(10)$ is used to determine the maximal element edge length in the tire/road contact area. In order to satisfy Nyquist's theorem for the given tire rolling speed of $22.22 \mathrm{~ms}^{-1}$ (i.e. $n_{\lambda_{r}}=2$ ), this corresponds to a maximal element edge length of $2.75 \cdot 10^{-2} \mathrm{~m}$. However, taking into account the contact conditions (i.e. the discretization of the contact surface needs to be fine enough), a maximum element edge length of $1.38 \cdot 10^{-2} \mathrm{~m}$ is used (i.e. $n_{\lambda_{r}}=4$ ). This yields a maximum excitation frequency of $800 \mathrm{~Hz}$.

Given the maximum frequency of interest, the minimal Nyquist sampling frequency should be around $1,000 \mathrm{~Hz}$ (the theoretical minimum is $800 \mathrm{~Hz}$ ). Therefore, the corresponding timestep $\Delta t$ as used for the simulations should be at least $10^{-3}$ seconds to capture the relevant dynamics. In order to meet the persistency condition (see section 2.4), a timestep $\Delta t$ equal to $10^{-4}$ seconds is chosen. This allows to keep the set of geometrical road surface constraints (sampled with a timestep of $10^{-3}$ seconds) constant for multiple subsequent timesteps.

Hyper-elastic constitutive models, such as the Neo-Hookean and MooneyRivlin models [27, Chapter 6.6], 28, Chapter 11.2] are used to model the rubber tire components. The reader is referred to the work by Helnwein et al. [39] for more information on typical material parameters. A Prony-series approach is used to model the viscous behavior of the rubber materials [40, Chapter 4.3]. 
All of the material properties are extracted from material sample measurements. The test tire design geometry (i.e. the construction design) is used to define the discretized numerical tire domain. The tire model is thus constructed a-priori, without the use of experimental test data of the real test tire (i.e. a-posteriori). This full order model (FOM) contains over 38,000 elements and 175,000 degrees of freedom (DOFs). The reader is referred to the work by Helnwein et al. [39], as well as [41, Section 3.1] for more information on the discretization of the 435 tire domain and/or tire models, as a similar discretization approach is used for the creation of the FOM in this work. The presented predictive approach can therefore also be applied to the tire models presented in [39] and [41, Section 3.1], or any other FE tire models.

The proposed fully predictive high-fidelity method is implemented in a fully nonlinear vibro-acoustic FE Matlab framework. Using the FOM and the coarse road surface scan, a hyper-reduced order model (HROM) is constructed. The relative size of the HROM with respect to the FOM is given in Table1. The total speedup-factor $(\mathrm{SF})$, shown in Table 1 , is the ratio of the total simulation time of the FOM and the total simulation time of the HROM. The latter also includes ${ }_{445}$ the setup time of the HROM (i.e. calculation of the ROB and reduced element sets), in order to have a fair and valid comparison. Both the FOM and HROM simulations use identical nodes of a high performance computing (HPC) cluster for their calculations, to again allow for fair comparison. For the simulation of one second of rolling, using $\Delta t=10^{-4}$ seconds, the SF is in the order of 450 a factor 100. This means in practice that for the implementation within the Matlab framework, simulation times are reduced from months to hours, making the proposed overall method feasible to use in a daily engineering practice 2

Table 1: Full order model (FOM) and hyper-reduced order model (HROM) properties

\begin{tabular}{ccccccc} 
& \# DOFs & $n_{\chi}$ & $n_{\Psi}$ & $|E|$ & $\left|E_{p}\right|$ & SF \\
\hline FOM & 176176 & - & - & 38376 & 6136 & 1 \\
\hline HROM & 1400 & 100 & 600 & 1400 & 700 & 73.33 \\
\hline
\end{tabular}

In order to investigate the benefit of using the proposed reduced fully nonlinear approach (i.e. the HROM), two alternative linearized approaches are defined as follows:

- Purely linear: only the contact force term $\mathbf{f}_{\mathbf{c}}$ and its tangent stiffness matrix $\mathbf{K}_{\mathbf{c}}$ are kept configuration-dependent. All of the other nonlinear configuration-dependent terms are linearized around a reference pressurized configuration. The static preloading of the tire onto the road surface

\footnotetext{
${ }^{2}$ Implementation of the proposed method into an optimized framework, i.e. written specifically for the host architecture, should reduce the absolute computational time values even more, while still retaining the same SF.
} 
is done using linear internal force and pressure force expressions. The ROB consists of linear constraint modes, i.e. calculated using the linear internal force and pressure force expressions, and eigenmodes calculated around a set of these linear constraint modes.

- Pseudo-linear: again the contact force term $\mathbf{f}_{\mathbf{c}}$ and its tangent stiffness matrix $\mathbf{K}_{\mathbf{c}}$ are kept configuration-dependent. Different from the purely linear approach is that the static preloading remains fully nonlinear. The ROB is identical to the one used for the fully nonlinear approach. After the preloading, all of the nonlinear configuration-dependent terms (except for the contact force terms) are linearized around a reference nonlinear static contact configuration and are kept linear throughout the dynamic simulation.

Here, the approaches start from having a fully nonlinear model available. Both approaches use linear internal force and pressure force expressions during the dynamic simulation. The reduced terms (23)-(27) therefore only need to be calculated once a-priori, where the internal force term $\mathbf{f}$ and pressure force term $\mathbf{f}_{\mathrm{p}}$ vanish for the purely linear approach and are constant for the pseudo-linear approach. No hyper-reduction thus needs to be applied, which allows for good speedup factors. A comparison of the approaches is made in Table 2 . Besides the total SF, the SF with respect to the fully nonlinear method is also given and is denoted as the relative speedup-factor (RSF).

Table 2: Comparison of the SFs and RSFs of the reduced fully nonlinear, linear and pseudolinear approaches

\begin{tabular}{ccc} 
& SF & RSF \\
\hline Nonlinear & 73.33 & 1 \\
\hline Linear & 792 & 10.8 \\
\hline Pseudo-linear & 330 & 4.55 \\
\hline
\end{tabular}

\subsection{Comparison of experimental and numerical results}

Using the test setup discussed above, hub force measurement data is extracted from the test tire rolling over the coarse road surface. The hub forces are measured at the spindle that holds the tire. This spindle shows no coupling effects with tire/rim assemblies below at least $500 \mathrm{~Hz}$, thus allowing for an indirect measurement of the hub forces. The numerical hub forces are calculated as explained in section 2. The same static vertical load is applied to the numerical tire model, and the axle height is kept locked throughout the simulations as well. The frequency spectra of the experimentally measured vertical hub force and 490 simulated vertical hub force are calculated using 1 second of time-domain data and a Fast Fourier Transform (FFT). A $50 \%$ overlap is used to reduce numerical artifacts and noise. At a sample rate of $10 \mathrm{kHz}$ (i.e. the timestep size of $10^{-4}$ seconds), this yields blocks of 5000 time-domain samples and a corresponding 
frequency resolution of $2 \mathrm{~Hz}$. Due to the specific case of steady-state rolling, spectral convergence is achieved for the frequency range of interest for both the experimental and numerical results. The vertical hub force spectra, calculated using the reduced fully nonlinear approach (HROM), linear approach and pseudo-linear approach are shown and compared with the experimental vertical hub force spectrum in Figure 11. It should be noted that normalized amplitudes 500 are shown, and therefore the ratio of amplitudes of frequencies of interest should be compared, rather than the difference between these normalized amplitudes.

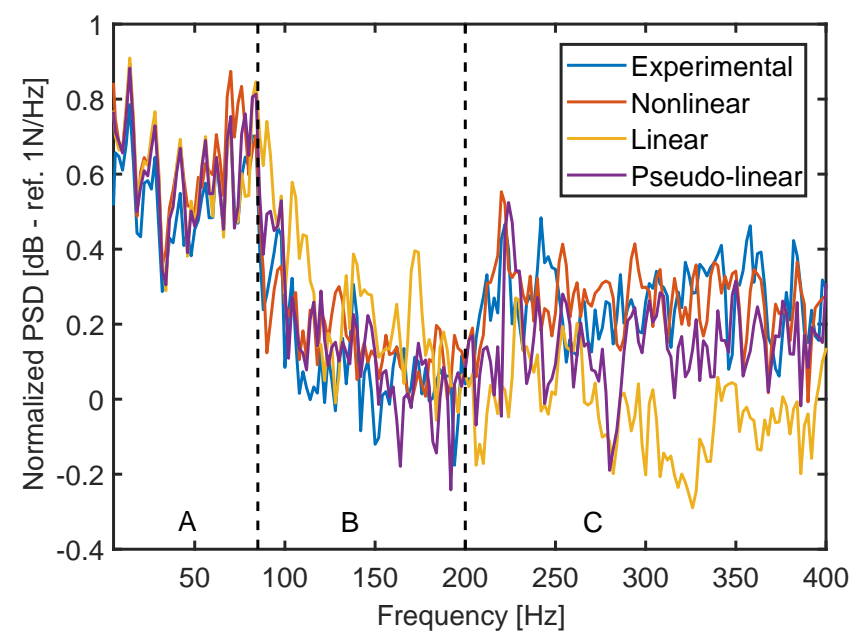

Figure 11: Comparison of the experimentally measured vertical hub force, shown in blue, and the simulated vertical hub force using the reduced fully nonlinear approach, shown in red, the linear approach, shown in orange, and the pseudo-linear approach, shown in purple.

The RMS-averaged amplitude difference of the simulated vertical hub force, using the three approaches, with respect to the experimental vertical hub force is shown in Figure 12. The RMS-averaged amplitude difference is calculated for 505 frequency bands with a $60 \mathrm{~Hz}$ bandwidth, in order to get a qualitative overview of the performance of each approach. 


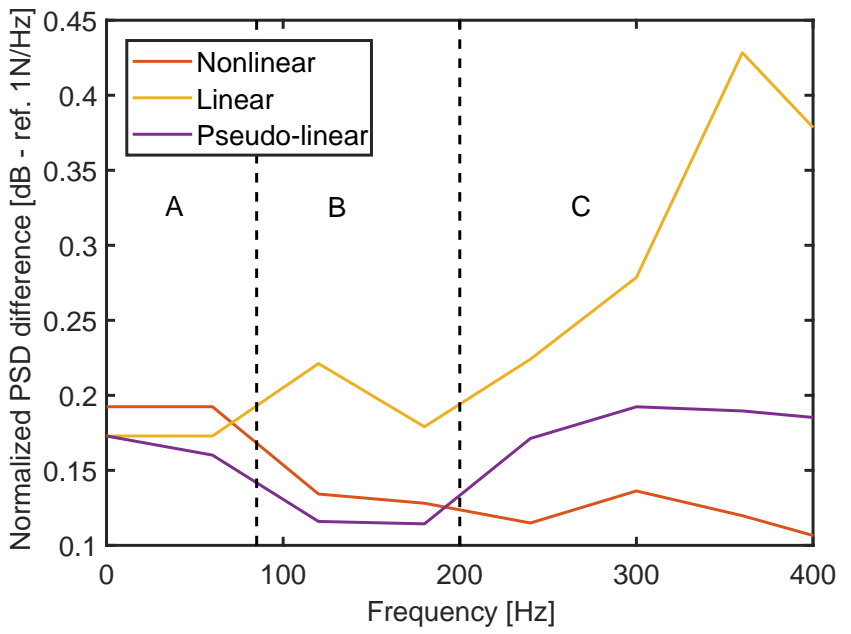

Figure 12: Comparison of the RMS-averaged hub force amplitude difference, calculated with respect to the experimental vertical hub force using the reduced fully nonlinear approach, shown in red, the linear approach, shown in orange, and the pseudo-linear approach, shown in purple

The frequency spectra of the total vertical contact forces (i.e. the summation over the vertical contact force components), as calculated using the reduced fully nonlinear, linear and pseudo-linear approaches are shown in Figure 13. The same FFT settings as for the hub force spectra, as shown in Figure 11, are used. Again normalized amplitudes are shown.

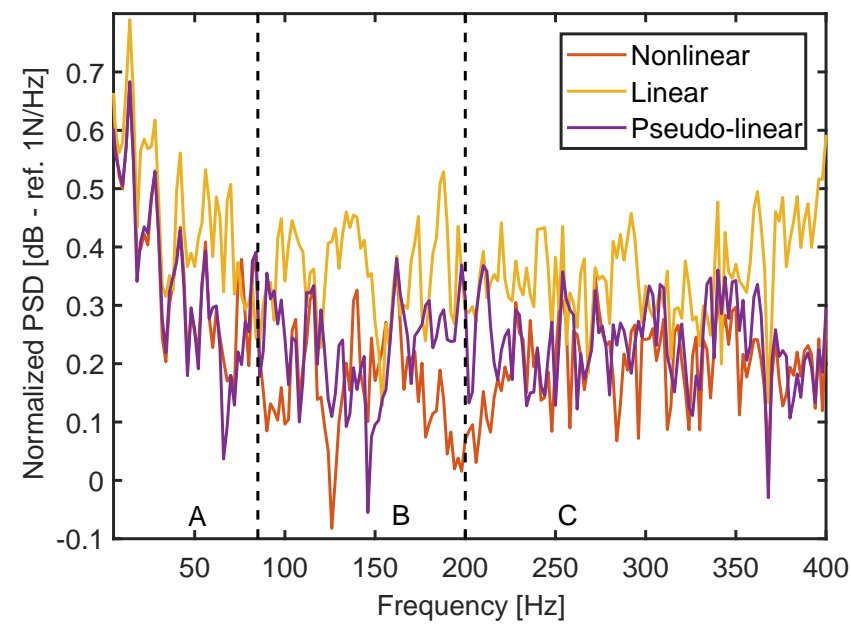

Figure 13: Comparison of the total vertical contact force as calculated using the reduced fully nonlinear approach, shown in red, the linear approach, shown in orange, and the pseudo-linear approach, shown in purple 
Based on the results shown in Figures 11 and 12, three frequency ranges, denoted as A, B and C, are defined. The properties of the frequency ranges are listed in Table 3

Table 3: Definition of the frequency ranges

\begin{tabular}{ccc} 
Frequency range & Start frequency $[\mathrm{Hz}]$ & End frequency $[\mathrm{Hz}]$ \\
\hline $\mathrm{A}$ & 0 & 85 \\
\hline $\mathrm{B}$ & 85 & 200 \\
\hline $\mathrm{C}$ & 200 & 400 \\
\hline
\end{tabular}

The comparison of the three approaches with the experimental results is further discussed per frequency range. In the following sections, the naming convention for tire modeshapes as introduced by Wheeler et al. 42. and extended by Kindt et al. 43 is used. Here, two indices $(c, a)$ are used which describe the bending order of the tire belt package in two directions. The first 520 index, $c$, represents the number of circumferential bending wavelengths. The second index, $a$, represents the number of half-wavelengths in the lateral (axial) direction of the belt, at a circumferential location where the shape is at an extreme radial displacement 43 .

\subsubsection{Frequency range A: $0-85 \mathrm{~Hz}$}

When looking at the static vertical preloading force and corresponding vertical hub displacement, as shown in Figure 14, a difference can be observed between the linear approach and the fully nonlinear and pseudo-linear approaches (where the latter are equivalent, as the pseudo-linear approach is also nonlinear during the static preloading). The initial tire/road contact area arising from the

530 static preloading is therefore not matched for the linear approach and the fully nonlinear and pseudo-linear approaches. Additionally, the vertical tire stiffness ${ }^{3}$, as shown in Figure 15, is higher for the linearized approach. This can be explained by the lack of the geometrical and rubber material nonlinearities during the static preloading, which typically yield lower stresses for the same 535 strains/displacements (i.e. more compliance). In order to correctly capture the static tire behavior and resulting tire/road contact area, nonlinear geometrical and material behavior therefore needs to be taken into account during static preloading. Looking at the total vertical contact force spectra, as shown in Figure 13 the linear approach yields higher amplitudes than the other approaches, which could be explained due to the overall tire stiffness being too high, and the corresponding local compliance being too low, resulting in larger contact forces in order to meet the imposed geometrical road surface constraints. However, when comparing the hub force spectra for this frequency range, where the $(1,0)$

\footnotetext{
${ }^{3}$ This vertical tire stiffness is defined as the increment in vertical loading force divided by the increment in vertical hub displacement
} 
structural mode of the tire tends to define the vertical hub force spectrum and structure-borne interior noise for the $60-80 \mathrm{~Hz}$ frequency range 1, 5 all three approaches perform to a similar level of accuracy. This could be explained by the fact that the influence of the $(1,0)$ structural mode on the vertical hub force (i.e. the transmittance of the contact forces to the hub) is less sensitive to the correct modeling of the tire/road contact interface, the modeling of the coarse 550 road excitation (i.e. local compliance), as well as the overall stiffness of the tire model. However, as discussed below, these effects do appear to be important for higher frequencies.

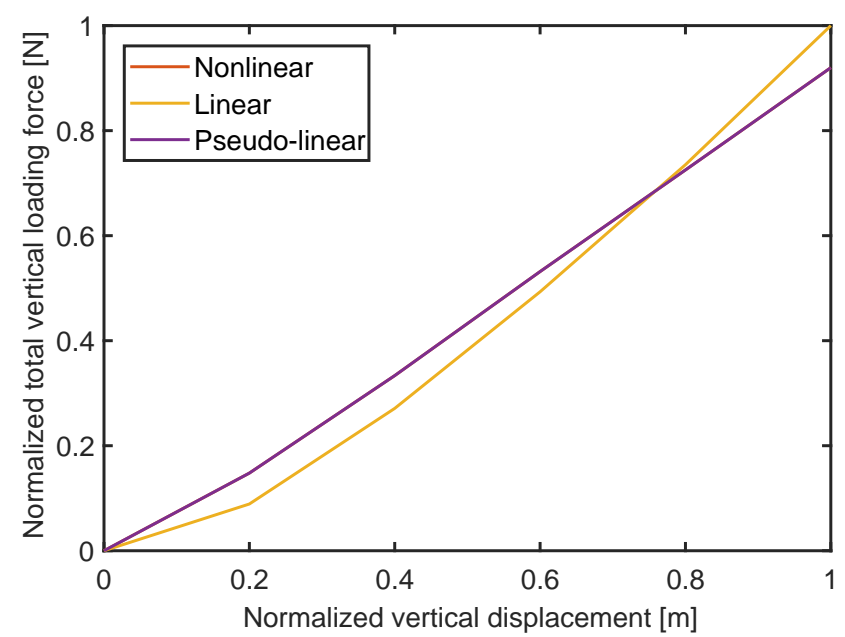

Figure 14: Comparison of the total vertical loading force and vertical displacement of the tire center (the hub) of the fully nonlinear approach, shown in red, the linear approach, shown in orange, and the pseudo-linear approach, shown in purple. 


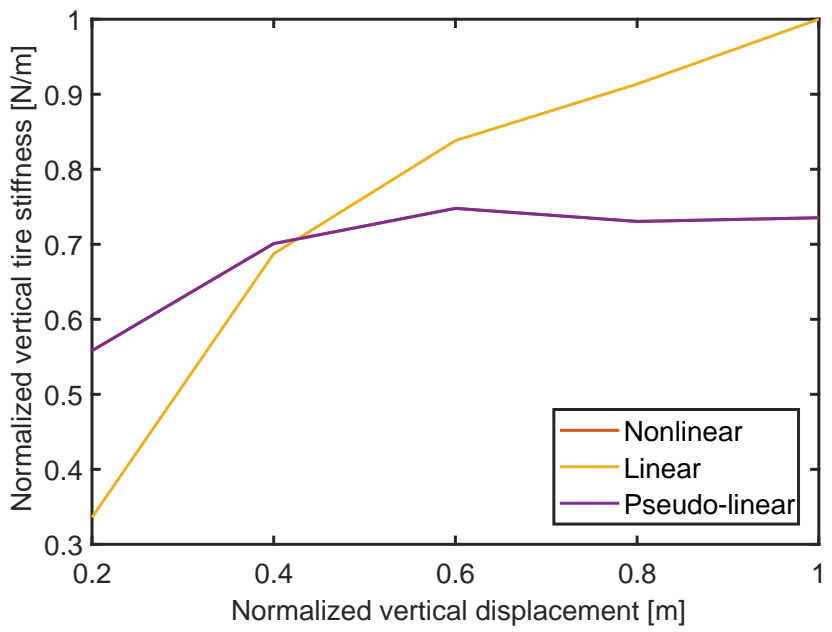

Figure 15: Comparison of the total vertical tire stiffness and vertical displacement of the tire center of the fully nonlinear approach, shown in red, the linear approach, shown in orange, and the pseudo-linear approach, shown in purple.

\subsubsection{Frequency range B: $85-200 H z$}

While the reduced fully nonlinear approach and the pseudo-linear approach show similar results for both the total vertical contact force spectra and hub force spectra, the linear approach clearly deviates from these results. Again, a possible explanation is that the tire/road contact area as calculated using the linear approach differs from the reduced nonlinear and pseudo-linear approaches, due to the linearized numerical tire model being too stiff. This yields both a different set of eigenmodes (due to the different contact area boundary conditions and larger stiffness of the linearized numerical tire model) and a different excitation of the tire (i.e. different contact forces arising for the linear approach when applying the same geometrical road surface constraints as for the other approaches). As a result, the linear approach also does not match 565 the experimental hub force results for the $85-200 \mathrm{~Hz}$ frequency range and up. A comparison of the tire/road contact area calculated using the three different approaches is shown in Figure 16. A snapshot is taken from the dynamic simulations at $t=0.1 \mathrm{~s}$, where the same constraints are applied to all three approaches. As can be observed, the tire/road contact area for the linear approach clearly 570 deviates from the reduced fully nonlinear approach and pseudo-linear approach, which could be attributed to the higher stiffness of the numerical tire model and the corresponding different excitation. A comparison of snapshots, taken at $t=0.1 \mathrm{~s}$ as well, from the tire/road contact force distributions of the three different approaches is shown in Figure 17. While it can be observed that both 575 the linear and pseudo-linear approaches have less contact force components than the reduced fully nonlinear approach, this becomes more clear when visualizing the difference between the reduced fully nonlinear approach and the linearized approaches. The difference in contact force components (i.e. the components 
not present for the linearized approaches) is shown in Figure 18. The linear

approach clearly has more missing contact force components than the pseudolinear approach, which again can be explained by the too large stiffness and missing local compliance of the linear tire model.

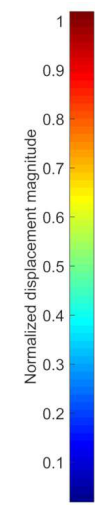

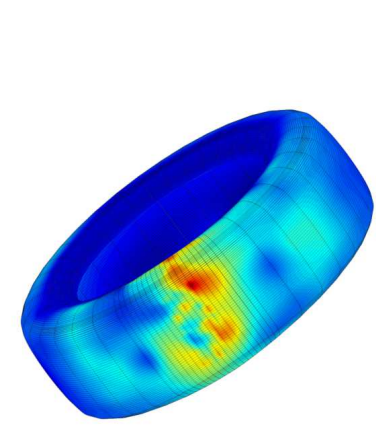

(a)

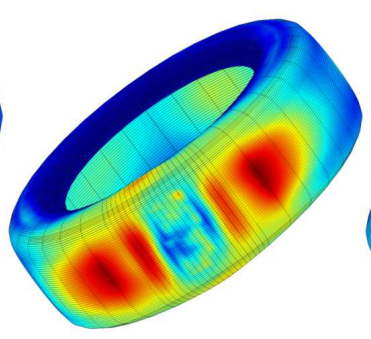

(b)

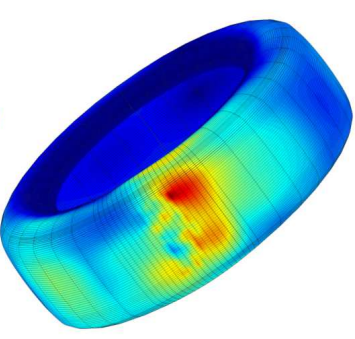

(c)

Figure 16: Comparison of the tire/road contact area for the reduced fully nonlinear approach (a), the linear approach (b) and the pseudo-linear approach (c), snapshot taken at $t=0.1 \mathrm{~s}$ 


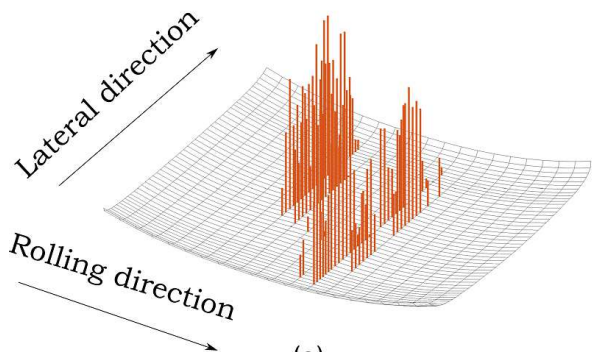

(a)

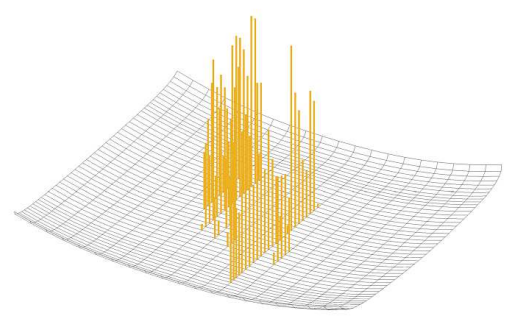

(b)

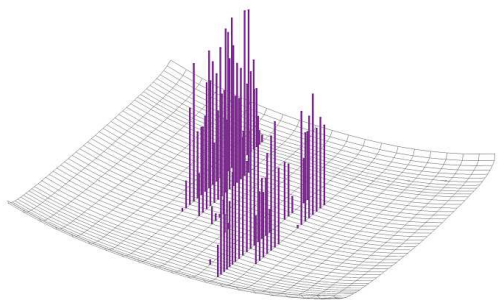

(c)

Figure 17: Comparison of the tire/road contact force distribution in the tire/road contact area for the reduced fully nonlinear approach (a), the linear approach (b) and the pseudo-linear approach (c), snapshot taken at $t=0.1 \mathrm{~s}$

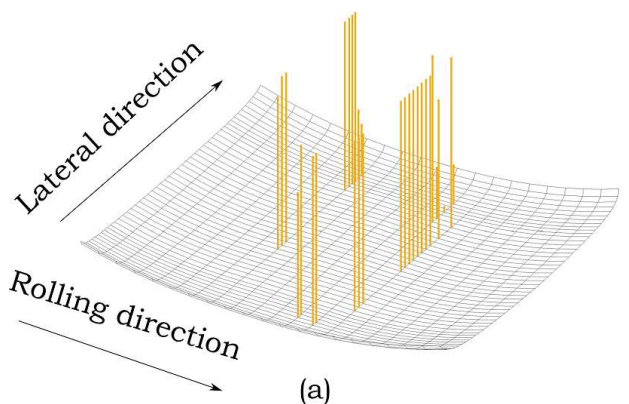

(a)

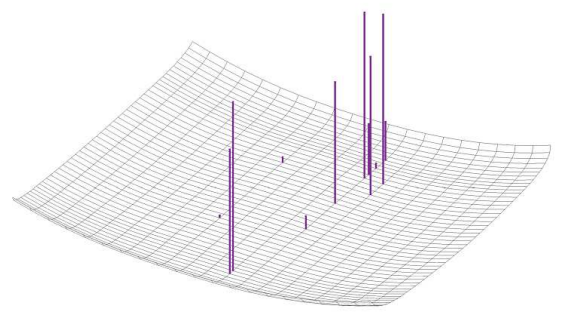

(b)

Figure 18: Difference between the the tire/road contact force distribution of the linear approach (a) and the pseudo-linear approach (b) with the reduced fully nonlinear approach, snapshot taken at $t=0.1 \mathrm{~s}$ 


\subsubsection{Frequency range C: 200-400Hz}

The acoustic cavity modes tend to dominate the $200-250 \mathrm{~Hz}$ zone of this fre-

585 coupling in the FE tire model. Again the linear approach clearly deviates from both the experimental results as well as the other approaches. Contrary to the other frequency ranges, the overall amplitude level of the hub force spectrum of the linear approach is now lower than those of the other approaches, remains, as before, higher than the other approaches. The amplitude difference regarding the hub forces could be attributed to the overall dynamics not being correctly captured by the linear approach, leading to a different transmittance of the contact forces to the hub (and corresponding hub forces). This can also linear approach. For the discussed frequency range, the pseudo-linear approach also deviates from the experimental results as well as the reduced fully nonlinear approach when comparing the hub force spectra. The overall amplitude level of the pseudo-linear hub force spectrum is, similar to the linear approach, lower than the reduced fully nonlinear approach for this frequency range. Comparison of the total vertical contact force spectra of the pseudo-linear and reduced fully nonlinear approaches shows that, again similar to the linear approach, the pseudo-linear approach now has a larger overall amplitude level. This could be explained by the fact that local nonlinear behavior of the tire tread in the 605 tire/road contact area (at the level of the road surface asperities) is not captured by the pseudo-linear approach, resulting in too stiff/non-compliant behavior and, as a result, too large contact force amplitudes and a different transmittance of the contact forces to the hub as well. This can also be observed from Figures 16- 18, where the pseudo-linear approach shows less local compliance (i.e. the missing contact force components) than the reduced fully nonlinear approach. The linear approach suffers from the same limitations, but at a larger scale than the local asperity-level, i.e. at tire/road contact area scale lengths.

\subsubsection{Conclusion}

The reduced fully nonlinear approach (i.e. the HROM) performs the best for the entire frequency range of interest, whereas the linear and pseudo-linear approach do not. For hub force prediction, the linear approach can be used for the $0-85 \mathrm{~Hz}$ frequency range, whereas the pseudo-linear approach can be used for the $0-200 \mathrm{~Hz}$ frequency range. For contact force simulation purposes, the linear approach cannot be used, whereas the pseudo-linear approach can be used for the $0-200 \mathrm{~Hz}$ range. Both linearized approaches lack the local compliant behavior of the tread surface, which leads to both different contact forces and a different vertical contact-to-hub force transmittance. The linear approach shows the missing compliance at tire/road contact area scale lengths, whereas the pseudolinear approach mainly shows this limitation at road surface asperity level/scale ${ }_{625}$ lengths. Therefore, the fully nonlinear approach remains the best choice for the frequency range of interest, even given the larger overall computational costs. A good correspondence between the experimental data and numerical data can 
be observed, especially given the fact that a purely predictive approach is used for the calculation of the numerical results.

One of the additional benefits of the predictive numerical approach is that the influence of a specific tire construction design on the transmitted contact forces, i.e. the hub forces, can be investigated. This allows to investigate e.g. the vertical contact-to-hub force transmissibility of a tire rolling over a coarse road surface, including all of the coupling effects that are missing when using a 635 non-rolling tire approach. For the experimental approach, the contact forces are unknown and thus the vertical contact-to-hub force transmissibility under rolling conditions cannot be measured or calculated. The contact force distribution for different tire designs under rolling conditions can be compared as well for the presented predictive approach, in order to investigate e.g. the influence of tire design parameters on the resulting contact forces. Again, this is not possible under rolling conditions for the experimental approach, where only hub forces can be used for comparison.

\section{Conclusion}

In this work, a fully predictive high-fidelity numerical approach for the calculation of dynamic tire/road rolling contact forces and corresponding dynamic hub forces is presented as an alternative to the experimental approaches. The proposed approach uses a fully nonlinear vibro-acoustic FE tire model in combination with an ALE formulation to describe both the rolling dynamics as well as the tire/road interaction. The tire/road interaction is modeled using a geometrical constraint formulation, rather than a constitutive modeling approach. Use of the geometrical constraint approach allows for the method to remain fully predictive, i.e. to not rely on measurement data.

A direct consequence of using the geometrical constraint approach in combination with a fully nonlinear vibro-acoustic tire FE model, is the large com655 putational cost of the numerical simulations. Therefore, in order to make the proposed approach computationally feasible, a nonlinear hyper-reduction technique, the MEM method, is applied. Application of the MEM method for the specific case of the simulation of one second of rolling over a coarse road surface, results in a speedup-factor in the order of a factor 100, effectively reducing the total simulation time from months to hours. In order to investigate the benefit of the proposed reduced fully nonlinear FE approach, two alternative linearized approaches are proposed and compared with both the experimental data and the reduced fully nonlinear approach simulation data. While these linearized approaches offer larger speedup-factors, they are not able to match 665 the experimental data across the entire frequency range of interest, unlike the reduced fully nonlinear approach. Therefore, the use of the reduced fully nonlinear approach is proposed, even though the associated computational cost is larger than the computational cost of the linearized approaches.

Comparison of experimental data from a tire rolling over a coarse road sur670 face and numerical simulation results from the equivalent hyper-reduced nonlinear vibro-acoustic FE tire model, shows that a good correspondence can be ob- 
served. The proposed predictive high-fidelity simulation approach could therefore be seen as an alternative for the time-consuming experimental approaches. Extending the frequency range and including e.g. high-fidelity suspension models in the proposed method are the possible subject of future work and research.

\section{Acknowledgments}

The research of Daniel De Gregoriis is funded by an AFR-PhD grant supported by the Fonds National de la Recherche, Luxembourg (AFR PhD Grant Agreement PhD 2015-1, Project Reference 10222097). The Fund for Scientific Research - Flanders (F.W.O.) is gratefully acknowledged for the support of the postdoctoral research of Frank Naets. The Industrial Research Fund KU Leuven is gratefully acknowledged for its support.

\section{References}

[1] S. Vercammen, Tyre/road noise due to road surface texture excitations: Experimental and numerical analyses, Ph.D. thesis, KU Leuven, Leuven, Belgium (2017).

[2] C. Hoever, The simulation of car and truck tyre vibrations, rolling resistance and rolling noise, Ph.D. thesis, Chalmers University of Technology, Göteborg, Sweden (2014).

[3] J. Winroth, Contact stiffness in tyre/road noise modelling and speed dependencies of tyre/road noise generation mechanisms, Ph.D. thesis, Chalmers University of Technology, Göteborg, Sweden (2017).

[4] W. R. Graham, Tyre rolling simulation using a state-space formulation, in: Proceedings of ISMA2014 including USD2014, 2014, pp. 1731-1746.

[5] P. Kindt, P. Sas, W. Desmet, Development and validation of a three-dimensional ring-based structural tyre model, Journal of

n. Sound and Vibration 326 (3) (2009) 852 - 869. doi:https: //doi.org/10.1016/j.jsv.2009.05.019.

11 URL http://www.sciencedirect.com/science/article/pii/ S0022460X0900457X

[6] W. Kropp, Structure-borne sound on a smooth tyre, Applied Acoustics

ㅁ 26 (3) (1989) 181 - 192. doi:https://doi.org/10.1016/0003-682X(89) 90051-0. $0003682 \times 89900510$

[ [7] K. Larsson, W. Kropp, A high-frequency three-dimensional tyre model based on two coupled elastic layers, Journal of Sound and Vibration 253 (4) (2002) 889 - 908. doi:https://doi.org/10.1006/jsvi.2001.4073. URL http://www.sciencedirect.com/science/article/pii/ S0022460X01940738 
[ [8] R. Pinnington, A wave model of a circular tyre. part 1: belt modelling, Journal of Sound and Vibration 290 (1) (2006) 101 - 132. doi:https://doi.org/10.1016/j.jsv.2005.03.023. URL http://www.sciencedirect.com/science/article/pii/ S0022460X05002634

n [9] R. Pinnington, A wave model of a circular tyre. part 2: side-wall and force transmission modelling, Journal of Sound and Vibration 290 (1) (2006) 133 - 168. doi:https://doi.org/10.1016/j.jsv.2005.03.024. URL http://www.sciencedirect.com/science/article/pii/ S0022460X05002646

[10] P. Sabiniarz, W. Kropp, A waveguide finite element aided analy-

a sis of the wave field on a stationary tyre, not in contact with the ground, Journal of Sound and Vibration 329 (15) (2010) 3041 - 3064. doi:https://doi.org/10.1016/j.jsv.2010.02.008.

725 uRL http://www.sciencedirect.com/science/article/pii/ S0022460X10001124

[11] M. Brinkmeier, U. Nackenhorst, S. Petersen, O. von Estorff,

1 A finite element approach for the simulation of tire rolling noise, Journal of Sound and Vibration 309 (1) (2008) 20 - 39.

730 doi:https://doi.org/10.1016/j.jsv.2006.11.040.

a URL http://www.sciencedirect.com/science/article/pii/ S0022460X07001198

[12] I. Lopez Arteaga, Green's functions for a loaded rolling tyre, International Journal of Solids and Structures 48 (25) (2011) 3462 - 3470. doi:https://doi.org/10.1016/j.ijsolstr.2011.09.006.

10 URL http://www.sciencedirect.com/science/article/pii/ S0020768311003064

[13] U. Nackenhorst, Finite element analysis of tires in rolling contact, GAMM-Mitteilungen 37 (1) (2014) 27-65. arXiv:https: //onlinelibrary.wiley.com/doi/pdf/10.1002/gamm.201410003, doi:10.1002/gamm.201410003.

a URL https://onlinelibrary.wiley.com/doi/abs/10.1002/gamm. 201410003

[14] P. Wriggers, Computational Contact Mechanics: Second Edition, SpringerVerlag, 2006.

[15] P. Andersson, W. Kropp, Time domain contact model for tyre/road interaction including nonlinear contact stiffness due to small-scale roughness, Journal of Sound and Vibration 318 (1) (2008) $296-312$. doi:https://doi.org/10.1016/j.jsv.2008.04.013. URL http://www.sciencedirect.com/science/article/pii/ S0022460X08003635 
[16] T. A. Laursen, Computational Contact and Impact Mechanics, SpringerVerlag, 2003.

[17] C. Hoever, W. Kropp, A model for investigating the influence of road surface texture and tyre tread pattern on rolling resistance, Journal of Sound and Vibration 351 (2015) $161-176$. doi:https://doi.org/10.1016/j.jsv.2015.04.009. URL http://www.sciencedirect.com/science/article/pii/ S0022460X15003417

[18] F. Wullens, W. Kropp, A three-dimensional contact model for tyre/road interaction in rolling conditions, Acta Acustica 90 (4) (2004) 702-711.

[19] W. Kropp, Ein Modell zur Beschreibung des Rollgeräusches eines unprofilierten Gürtelreifens auf rauher Straßenoberfläche, number 166 in Fortschrittberichte VDI, Reihe 11: Schwingungstechnik, VDI-Verlag, 1992.

$765[20]$ J. Winroth, P. Andersson, W. Kropp, Importance of tread inertia and damping on the tyre/road contact stiffness, Journal of

n. Sound and Vibration 333 (21) (2014) 5378 - 5385. doi:https: //doi.org/10.1016/j.jsv.2014.05.030.

URL http://www.sciencedirect.com/science/article/pii/ S0022460X14004398

[21] K. Larsson, S. Barrelet, W. Kropp, The modelling of the dynamic behaviour of tyre tread blocks, Applied Acoustics 63 (6) (2002) $659-677$. doi:https://doi.org/10.1016/S0003-682X(01)00059-7. URL http://www.sciencedirect.com/science/article/pii/ S0003682X01000597

[22] U. Nackenhorst, The ALE-formulation of bodies in rolling contact: Theoretical foundations and finite element approach, Computer Methods in Applied Mechanics and Engineering 193 (39) (2004) 4299 - 4322. doi:https://doi.org/10.1016/j.cma.2004.01.033. URL http://www.sciencedirect.com/science/article/pii/ S0045782504002233

[23] F. Naets, W. Desmet, A-priori element selection and weighting for hyperreduction in nonlinear structural dynamics, MODRED 2017 3rd Workshop on model reduction of complex dynamical systems, Odense, Denmark (1113 January 2017).

[24] F. Naets, D. De Gregoriis, W. Desmet, Multi-expansion modal reduction: a A pragmatic semi-a priori model order reduction approach for nonlinear structural dynamics, International Journal for Numerical Methods in Engineering 0 (0). arXiv:https://onlinelibrary.wiley.com/doi/pdf/10. 1002/nme.6034, doi:10.1002/nme.6034.

URL https://onlinelibrary.wiley.com/doi/abs/10.1002/nme.6034 
[25] D. De Gregoriis, F. Naets, P. Kindt, W. Desmet, A nonlinear a-priori hyperreduction method for the dynamic simulation of a car tire rolling over a rough road surface, in: Proceedings of the Sixth European Conference on Computational Mechanics, Glasgow, UK, 2018, pp. 2430-2441.

[26] C. Farhat, P. Avery, T. Chapman, J. Cortial, Dimensional reduction of nonlinear finite element dynamic models with finite rotations and energybased mesh sampling and weighting for computational efficiency, International Journal for Numerical Methods in Engineering 98 (9) (2014) 625-662. arXiv:https://onlinelibrary.wiley.com/doi/pdf/10.1002/ nme.4668, doi:10.1002/nme.4668.

URL https://onlinelibrary.wiley.com/doi/abs/10.1002/nme.4668

[27] K.-J. Bathe, Finite Element Procedures, Prentice Hall, Inc., 1996.

[28] R. de Borst, M. A. Crisfield, J. J. Remmers, C. V. Verhoosel, Non-linear Finite Element Analysis of Solids and Structures: Second Edition, John Wiley \& Sons, Ltd, 2012.

[29] O. C. Zienkiewicz, R. L. Taylor, J. Z. Zhu, The Finite Element Method: its Basis and Fundamentals, 7th edition, Butterworth-Heineman, 2013.

[30] G. Everstine, Finite element formulatons of structural acoustics problems, Computers \& Structures 65 (3) (1997) 307 - 321. doi:10.1016/S0045-7949(96)00252-0.

a URL http://www.sciencedirect.com/science/article/pii/ S0045794996002520

[31] S. W. Rienstra, A. Hirschberg, An Introduction to Acoustics, Technische Universiteit Eindhoven, Eindhoven, 2004.

[32] U. Sandberg, J. A. Ejsmont, Tyre/Road Noise Reference Book, Informex, SE-59040 Kisa, Sweden, 2002.

[33] M. Ziefle, U. Nackenhorst, Numerical techniques for rolling rubber wheels: treatment of inelastic material properties and frictional con820 tact, Computational Mechanics 42 (3) (2008) 337-356. doi:10.1007/ s00466-008-0243-9.

URL https://doi.org/10.1007/s00466-008-0243-9

[34] A. Suwannachit, U. Nackenhorst, R. Chiarello, Stabilized numerical so1. lution for transient dynamic contact of inelastic solids on rough sur825 faces, Computational Mechanics 49 (6) (2012) 769-788. doi:10.1007/ s00466-012-0722-x.

URL https://doi .org/10.1007/s00466-012-0722-x

[35] M. Arnold, O. Brüls, Convergence of the generalized- $\alpha$ scheme for constrained mechanical systems, Multibody System Dynamics 18 (2) (2007)

185-202. doi:10.1007/s11044-007-9084-0

URL https://doi.org/10.1007/s11044-007-9084-0 
[36] A. van de Walle, F. Naets, E. Deckers, W. Desmet, Stability-preserving

- model order reduction for time-domain simulation of vibro-acoustic FE models, International Journal for Numerical Methods in Engineering

$835 \quad 109(6)(2017)$ 889-912. arXiv:https://onlinelibrary.wiley.com/doi/ pdf/10.1002/nme.5323, doi:10.1002/nme.5323.

URL https://onlinelibrary.wiley.com/doi/abs/10.1002/nme.5323

[37] R. R. Craig, A. J. Kurdila, Fundamentals of structural dynamics, 2nd Edition, Wiley, Hoboken, 2006.

[38] B. Blockmans, Model reduction of contact problems in flexible multibody dynamics, Ph.D. thesis, KU Leuven, Leuven, Belgium (2018).

[39] P. Helnwein, C. Liu, G. Meschke, H. Mang, A new 3-d finite element model for cord-reinforced rubber composites - application to analysis of automobile tires, Finite Elements in Analysis and Design 14 (1) (1993) 1 - 16. doi:https://doi.org/10.1016/0168-874X(93) 90075-2.

II URL http://www.sciencedirect.com/science/article/pii/ 0168874 X93900752

[40] O. C. Zienkiewicz, R. L. Taylor, D. D. Fox, The Finite Element Method for Solid and Structural Mechanics, 7th edition, Butterworth-Heineman, 2013.

[41] Dassault Systèmes Simulia, Abaqus Example Problems Manual, Dassault Systèmes.

[42] R. L. Wheeler, H. R. Dorfi, B. B. Keum, Vibration modes of radial tires: Measurement, prediction, and categorization under different boundary and operating conditions, in: SAE Technical Paper, SAE International, 2005. doi: $10.4271 / 2005-01-2523$ URL https://doi.org/10.4271/2005-01-2523

[43] P. Kindt, P. Sas, W. Desmet, Measurement and analysis of rolling tire vibrations, Optics and Lasers in Engineering 47 (3) (2009) 443 - 453, optical Measurements. doi:https://doi.org/10.1016/j.optlaseng. 2008.06 .017

URL http://www.sciencedirect.com/science/article/pii/ S0143816608001516 